\title{
Eye-Tracking and Economic Theories of Choice Under Risk
}

\author{
by
}

\author{
Glenn W. Harrison and J. Todd Swarthout ${ }^{\dagger}$
}

December 2018

\begin{abstract}
.
We examine the ability of eye movement data to help understand the determinants of decision making over risky prospects. We start with structural models of choice under risk, and use that structure to inform what we identify from the use of process data in addition to choice data. We find that information on eye movements does significantly affect the extent and nature of probability weighting behavior. Our structural model allows us to show the pathway of the effect, rather than simply identifying a reduced form effect. This insight should be of importance for the normative design of choice mechanisms for risky products. We also show that decision response duration is no substitute for the richer information provided by eye-tracking.
\end{abstract}

† Department of Risk Management \& Insurance and Center for the Economic Analysis of Risk, Robinson College of Business, Georgia State University, USA (Harrison); and Department of Economics, Andrew Young School of Policy Studies, Georgia State University, USA (Swarthout). Harrison is also affiliated with the School of Economics, University of Cape Town. E-mail contacts: gharrison@gsu.edu and swarthout@gsu.edu. 


\section{Table of Contents}

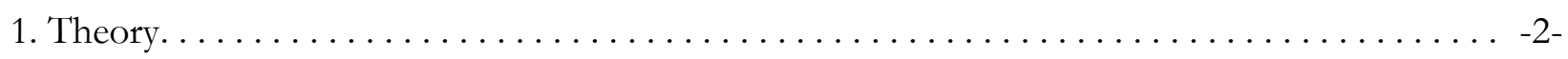

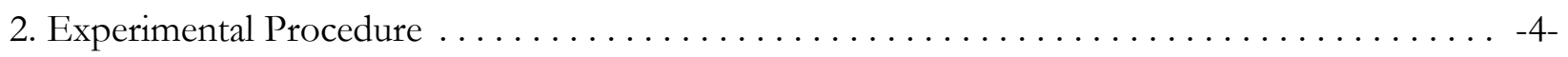

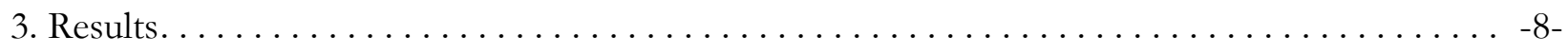

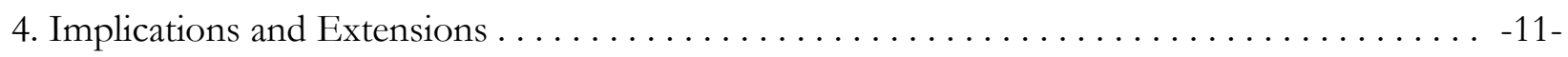

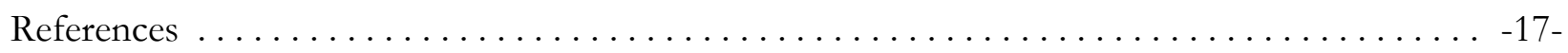

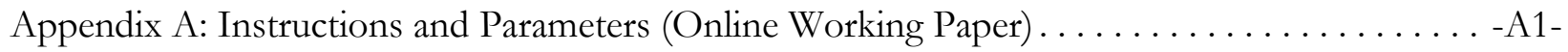

A. Introductory Text . . . . . . . . . . . . . . . . . .

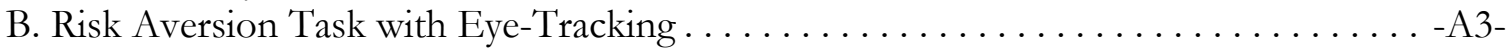

C Lotteries for the Standard Risk Aversion Task with No Eye-Tracking . . . . . . . . . . -A6-

Appendix B: Estimating Structural Models of Decision-Making (Online Working Paper) . . . . . -A9-

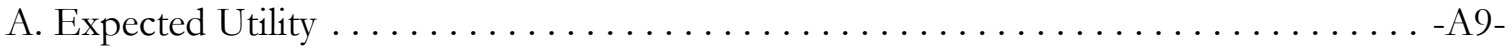

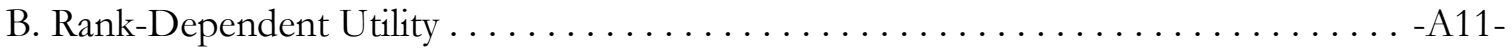

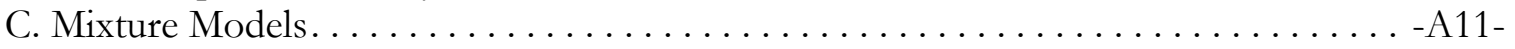

Appendix C: Previous Literature (Online Working Paper) $\ldots \ldots \ldots \ldots \ldots \ldots \ldots \ldots \ldots \ldots \ldots$. A13-

Appendix D: Detailed Estimates (Online Working Paper) $\ldots \ldots \ldots \ldots \ldots \ldots \ldots \ldots \ldots \ldots \ldots$. A20- 
Economic theories of choice under risk are mathematical characterizations of the outcome of some evaluation and decision-making process. Although expository stories about this process are told to rationalize the characterization, from a formal perspective they are irrelevant: it is only "as if" the individual followed this or that process. Since these theories are characterizations of optimization problems, the manner in which we analysts might numerically solve them provides "algorithmic" insight into a possible process that might have been followed, particularly when the algorithm is modeled as a path-following, homotopy algorithm (Harrison [2008; §4]). But there are literally infinite variations on these and other algorithms that can lead to the same optimization outcome, so at best this approach provides suggestive hypotheses about how decisions are made.

One role for process data is to inform our knowledge of "behaviorally plausible" decisionmaking algorithms that might be used. A related role might be to suggest descriptive explanations as to why some individuals tend to weight probabilities in the Rank Dependent Utility (RDU) sense. Finally, identification of the subset of information that is not studied, in absolute or relative terms, should provide insights for the normative evaluation of choices under risk.

Decision-process data can be collected in many ways, and we consider eye-tracking. Eyetracking provides a relatively non-intrusive method to collect rich data on areas of a visual display that are gazed upon. Mouse-tracking provides an alternative which involves software to mask the entirety of a visual display except for an unmasked region defined proximately by the instantaneous position of the mouse pointer, and yields sequences of information acquisition roughly analogous with eye-tracking. However, behavior observed with these two tracking methods differs systematically (Lohse and Johnson [1996]). Time-tracking is the least intrusive, and easy to program, but only provides one scalar for the entire process, without discriminating between areas of the visual display.

Our approach differs from previous literature by starting with formal economic theories of 
choice under risk, and then asking if eye-tracking data informs the statistical, structural characterization of choices. Previous research posits certain patterns of visual attention that are loosely derived from alternative structural models of choice under risk, but does not then relate the visual attention data to any structural econometric representation of that model. ${ }^{1}$ Just as one might add demographic covariates as linear functions of the structural parameters of a model of choice under risk when pooling over choices and subjects (Harrison and Rutström [2008; \2.2, §F.1]), we propose adding eye-tracking covariates as linear covariates when pooling over choices of a given subject.

We demonstrate this methodological approach by collecting data from subjects facing lottery choices with incentives while an eye-tracker is recording eye movements. We then estimate a structural model of the decision making of these subjects in which we allow for a mixture of Expected Utility Theory (EUT) and RDU theory. We demonstrate that information on eye movements significantly informs our characterization of preferences, particularly the RDU fraction of choices. This insight comes in a structural form: we can say more than that "eye movement data matters for choice." Moreover, we show that the total duration of time spent on the choice cannot substitute for the richer eye movement data.

\section{Theory}

Consider parametric versions of EUT and RDU models of decision making over objective probabilities, to introduce notation and basic concepts. Nothing hinges on the parametric assumptions, although the parametric forms assumed are standard in the literature. Assume that utility of income $\mathrm{x}$ is

\footnotetext{
${ }^{1}$ Appendix C reviews relevant literature in detail. Rosen and Rosenkoetter [1976] appears to be the first eye-tracking study of choice over risky lotteries, and initiated a theme that dominates later literature. Their motivation was to determine if eye movements over risky lotteries were "holistic" or "dimensional." Fiedler and Glöckner [2012] and Glöckner, Fiedler, Hochman, Ayal and Hilbig [2012] appear to be the first eye-tracking studies to provide salient rewards to lottery choice.
} 
defined by

$$
\mathrm{U}(\mathrm{x})=\mathrm{x}^{(1-\mathrm{r})} /(1-\mathrm{r})
$$

where $r \neq 1$ is a parameter to be estimated. Thus $r$ is the coefficient of Constant Relative Risk Aversion (CRRA) for an EUT individual: $r=0$ corresponds to risk neutrality, $r<0$ to risk loving, and $r>0$ to risk aversion.

Let there be J possible outcomes in a lottery defined over objective probabilities. Under EUT the probabilities for each outcome $\mathrm{x}_{\mathrm{j}}, \mathrm{p}\left(\mathrm{x}_{\mathrm{j}}\right)$, are those that are induced by the experimenter, so expected utility (EU) is simply the probability weighted utility of each outcome in each lottery i:

$$
\mathrm{EU}_{\mathrm{i}}=\sum_{\mathrm{j}=1, \mathrm{~J}}\left[\mathrm{p}\left(\mathrm{x}_{\mathrm{j}}\right) \times \mathrm{U}\left(\mathrm{x}_{\mathrm{j}}\right)\right]
$$

The RDU model of Quiggin [1982] extends the EUT model by allowing for decision weights on lottery outcomes. The specification of the utility function is the same parametric specification (1) considered for EUT; we just replace $\mathrm{r}$ with $\varrho$. To calculate decision weights $\mathrm{w}(\cdot)$ under RDU one replaces EU defined by (2) with RDU:

$$
\mathrm{RDU}_{\mathrm{i}}=\sum_{\mathrm{j}=1, \mathrm{~J}}\left[\mathrm{w}\left(\mathrm{p}\left(\mathrm{x}_{\mathrm{j}}\right)\right) \times \mathrm{U}\left(\mathrm{x}_{\mathrm{j}}\right)\right]=\sum_{\mathrm{j}=1, \mathrm{~J}}\left[\mathrm{w}_{\mathrm{j}} \times \mathrm{U}\left(\mathrm{x}_{\mathrm{j}}\right)\right]
$$

where

$$
\mathrm{w}_{\mathrm{j}}=\omega\left(\mathrm{p}_{\mathrm{i}}+\ldots+\mathrm{p}_{\mathrm{J}}\right)-\omega\left(\mathrm{p}_{\mathrm{i}+1}+\ldots+\mathrm{p}_{\mathrm{J}}\right)
$$

for $j=1, \ldots, J-1$, and

$$
\mathrm{w}_{\mathrm{j}}=\omega\left(\mathrm{p}_{\mathrm{j}}\right)
$$

for $\mathrm{j}=\mathrm{J}$, with the subscript $\mathrm{j}$ ranking outcomes from worst to best, and $\omega(\cdot)$ is some probability weighting function (pwf). The pwf proposed by Prelec [1998] exhibits considerable flexibility. This function is

$$
\omega(\mathrm{p})=\exp \left\{-\eta(-\ln \mathrm{p})^{\varphi}\right\}
$$

and is defined for $0<p \leq 1, \eta>0$ and $\varphi>0$. 
In the usual manner, we employ a Fechner noise parameter $\mu$ to adjust the strength with which the latent index favoring one lottery over the other lottery translates into the probability of observing that lottery being chosen. This specification is a part of the economics of the model, and is distinct from the use of a cumulative density function linking the latent index and the implied probability of choice of one lottery over the other.

Since we focus on the behavior of a representative agent, for simplicity, we consider the possibility that behavior is characterized as mixture of EUT and RDU decision-making. Following Harrison and Rutström [2009], let $\pi$ denote the probability that a given choice is made consistently with EUT, so that $1-\pi$ is the probability that the choice is made consistently with RDU. Maximum likelihood estimates of the parameters $r, \varrho, \eta, \varphi, \mu$ and $\pi$ can be obtained from the observed data, and each parameter can, in principle, be estimated as a linear function of observable demographic characteristics and eye-tracking characteristics. ${ }^{2}$

\section{Experimental Procedure}

Twenty undergraduate students participated in the experiment, and all had normal or corrected-to-normal vision. Each participant arrived at the lab and provided informed consent before starting the instructions, which were presented in both video and print formats. ${ }^{3}$ At the conclusion of video instructions, an experimenter would answer any questions. The participant then moved from the sign-in room to the eye-tracking room, and went through the eye-tracker calibration procedure, followed by 50 lottery choices, and finally one of the prior lottery choices was randomly selected and

${ }^{2}$ Appendix B sets out the formal econometric model.

3 Appendix A provides all printed instructions and links to video instructions. We use video to minimize session-specific variation. 
played for cash. No other task was conducted during the session. ${ }^{4}$ Each session lasted about 30 minutes and consisted of one participant. Subjects also received a non-salient $\$ 5$ for participating in the study, as well as expected salient earnings.

We aimed to minimize the likelihood that participants engage in covert attention of lottery information, as eye-tracking inferences are critically predicated upon overt attention. ${ }^{5}$ If systematic covert attention is present in an eye-tracking experiment, then arguably experimental control is compromised. Unfortunately, many of the prior eye-tracking experiments of choices over lotteries were designed to require covert attention. Rosen and Rosenkoetter [1976] presented each lottery with two outcomes, but only one probability was displayed while the other was implicit. Russo and Dosher [1983], Arieli, Ben-Ami and Rubinstein [2011] and Stewart, Hermens and Matthews [2016] all utilized two-outcome lotteries, but displayed only one outcome prize and associated probability per lottery, with the non-displayed outcome having a prize of $\$ 0$ and an implied probability. Janowski [2012; chapter II] used lottery pairs in which one lottery displayed a positive and negative outcome with an implicit third outcome of $\$ 0$ and an associated implicit probability, while the alternative lottery was always $\$ 0$ with certainty and not even displayed. Presenting lotteries with implicit information necessarily requires covert attention of the missing information, and so we opted to explicitly display all lottery information.

Additionally, all prior studies of which we are aware made use of a fixed number of lottery

\footnotetext{
${ }^{4}$ All participants belonged to a longitudinal panel and had participated in an experiment several months prior in which we collected demographics information and elicited risk preferences with a "traditional" display screen and no eye-tracking. The display is illustrated in Harrison, Hofmeyr, Ross and Swarthout [2018; Figure 1, p. 329], albeit with South African Rand denominations.

${ }^{5}$ Generally speaking, overt visual attention is the alignment of mental attention and visual sensory stimuli, while covert attention is the nonalignment of mental attention and visual stimuli (Posner [1980]). Overt-covert attention and the Eye-Mind hypothesis (Just and Carpenter [1980]) have been topics of considerable debate in the literature, and a review is beyond our scope here. The general consensus is that there is a strong linkage between eye movements and mental attention. For reviews and discussions of the experimental evidence in this debate, see Rayner [1998][2009] and Orquin and Loose [2013].
} 
outcomes across trials. All but one employed lotteries with invariantly two outcomes, ${ }^{6}$ while Janowski [2012; chapter II] presented lottery pairs consisting of one degenerate lottery and one three-outcome lottery. We opted instead for a variable number of lottery outcomes, both within and across lottery pairs, in order to encourage overt attention of all lottery information.

We also aimed to minimize the likelihood that participants would have an incentive to recall lottery information, as this could reduce the need of participants to acquire visual information. For example, if lotteries always have 50-50 odds, then participants may likely recall this fact in later trials and stop visually fixating on probability information displayed on screen. Similarly, if lotteries are displayed such that either monetary or probability values are ordered on-screen, then participants may begin inferring (and not visually attending to) information based on screen layout.

We constructed the lottery pairs for the experiment with several criteria in mind. First, we created one million individual lotteries, such that each lottery consisted of a uniformly random number of outcomes between one and four, each outcome probability was drawn uniformly with replacement from $\{5 \%, 10 \%, \ldots 100 \%\}$, subject to a constraint that they sum to $100 \%$, and each outcome amount was drawn uniformly without replacement from $\{\$ 0, \$ 1, \ldots \$ 100\}$. Second, from these one million lotteries, we formed 200 lottery pairs, such that within each pair the difference between the expected values (EVs) of the two lotteries was less than $\$ 5$, the lottery with the larger EV also had larger standard deviation of monetary amounts, ${ }^{7}$ and neither lottery stochastically dominated the other. Third, from these candidate 200 lottery pairs, 400 sets of 50 lottery pairs were randomly formed, and for each set we calculated EUT choice predictions based on CRRA values of $0.2,0.4,0.6$ and 0.8 , calculated the

\footnotetext{
${ }^{6}$ See Rosen and Rosenkoetter [1976], Russo and Dosher [1983], Arieli, Ben-Ami and Rubinstein
} [2011], Glöckner and Herbold [2011], Fiedler and Glöckner [2012], Glöckner, Fiedler, Hochman, Ayal and Hilbig [2012], Su, Rao, Hong-Yue, Du, Li and Li [2013] and Stewart, Hermens and Matthews [2016].

7 This property rewards in expectation a premium for taking on additional risk. Since the standard deviation formula is defined only over vectors of length greater than 1 , degenerate lotteries were assigned a standard deviation value of 0 . 
correlations between the 4 predictions, and ultimately selected the set of 50 lottery pairs that yielded the lowest average correlation, providing us the set of lotteries with the best discriminatory power (albeit subject to assumed functional form). The resulting lotteries used in this experiment are reported in Table A1 of Appendix A.

Figure 1 is a screen capture of the lottery display format, specifically pair \#48. The random lottery creation process resulted in generally unordered probability and monetary values across outcomes of a given lottery, and this lack of order was preserved in the ultimate visual display of the lotteries. In the event that a lottery had less than four outcomes, then the outcomes are displayed in a vertically contiguous manner and the vertical positioning is randomly determined (e.g., if a lottery has two outcomes, then there is a $1 / 3$ chance that the two outcomes are displayed in the first and second rows of the lottery, a $1 / 3$ chance the outcomes are displayed in the second and third rows, and a $1 / 3$ chance the outcomes are in the bottom two rows). The visual layout of each of the 50 lottery pairs is held constant across participants, while the order of the pairs is randomized for each participant.

Eye movements were captured with an Eyelink 1000 Plus system, manufactured by SR Research. We sampled eye movements at a rate of $1000 \mathrm{~Hz}$ and used a chin rest to reduce sampling noise due to head movement. Visual stimuli were displayed on a BenQ XL2411Z display monitor at a resolution of $1920 \times 1080$ at a distance of approximately $90 \mathrm{~cm}$. A nine-point calibration routine was conducted to insure average accuracy within one-half degree of visual angle and maximum error less than one degree. A drift check routine was conducted before each trial to insure accuracy was maintained across trials. Lottery prize and probability values were displayed numerically in 40 point Times New Roman font, and each non-overlapping interest area (IA) was defined by a rectangle of pixels 198 high by 248 wide centered on a given numeric value, resulting in each IA subtending visual angles of approximately 3.5 degrees vertical and 4.4 degrees horizontal. The raw data from the eye-tracker consists of x-y screen coordinates of gaze position - one per millisecond. From these temporal raw data, saccades are 
identified whenever gaze position velocity is greater than $30^{\circ} / \mathrm{sec}$ or acceleration is greater than $8000^{\circ} / \mathrm{sec}^{2}$, and the remaining gaze positions not belonging to saccades are identified residually as fixations. These fixations are typically located within one of the IA regions, and for each IA within a trial we define the IA's dwell time as the amount of time, in milliseconds, of all fixations that are located within the given IA. We next calculate the probability (prize) dwell time within a trial as the sum of dwell times of IAs associated with probabilities (prizes). And finally we transform these dwell times into proportions by normalizing with the sum of all dwell times.

After completing eye-tracker calibration, the participant began the decision trials. At the beginning of each trial, a fixation target was displayed in the center of an otherwise blank screen. The trial progressed to a lottery pair decision screen only if the system determined that the participant's gaze position was within two degrees visual angle of the position of the target. Once a pair of lotteries was displayed, the participant was afforded as much time as desired to submit a decision, and the decision was entered by pushing either the left or right button on a two-button button box; ${ }^{8}$ no keyboard or mouse was used during the decision trials. After the 50 trials were completed the participant rolled dice to randomly select a prior choice and realize a monetary outcome. ${ }^{9}$

\section{Results}

If we assume that EUT characterizes all choices, the CRRA parameter $\mathrm{r}$ is estimated to be 0.50 (95\% confidence $0.44 \leftrightarrow 0.57) .{ }^{10}$ If we assume that RDU characterizes all choices, we estimate $\varrho=0.2$ $(0.01 \leftrightarrow 0.41), \eta=1.17(0.93 \leftrightarrow 1.41)$ and $\varphi=0.56(0.43 \leftrightarrow 0.69)$. Thus RDU exhibits less diminishing marginal utility than EUT, and on balance generates the rest of the risk premium from probability

\footnotetext{
${ }^{8}$ Manufactured by Black Box Toolkit. see https://www.blackboxtoolkit.com.

${ }^{9}$ Precise details are provided in Appendix A.

${ }^{10}$ Appendix D documents all statistical results.
} 
optimism. ${ }^{11}$ The top two panels of Figure 2 display the estimated pwf and the implied decision weights, calculated for equi-probable reference lotteries with 2, 3 or 4 prizes in order to see the pure effect of probability weighting. ${ }^{12}$

When we allow a mixture of EUT and RDU to characterize choices, we estimate the proportion of RDU choices to be $0.56(0.36 \leftrightarrow 0.75)$, which is roughly consistent with the fraction that is obtained when estimating at the level of the individual for samples drawn from the same population (Harrison and $\mathrm{Ng}$ [2016; Figure 5, p. 103]). The striking effect of estimating the mixture model is that the utility function for RDU becomes linear: $\varrho=0.05(-0.19 \leftrightarrow 0.28)$. The EUT utility function remains roughly the same: $r=0.47(0.23 \leftrightarrow 0.72)$; and the RDU pwf remains about the same: $\eta=1.25(1.00 \leftrightarrow 1.50)$ and $\varphi=$ $0.52(0.39 \leftrightarrow 0.65)$. The bottom panels of Figure 2 show how similar the mixture pwf is to the case in which RDU characterized $100 \%$ of the choices rather than just $56 \%$ of the choices.

With these risk preferences as background, the effect of eye movements can be easily identified. We consider the percent of time spent dwelling on the probabilities, normalized by the time spent dwelling on prizes or probabilities (there was negligible time spent looking elsewhere). We add this covariate to a constant term for each of the $\varrho, \eta, \varphi, \mu$ and $\pi$ parameters. ${ }^{13}$ Since we do not control (yet) for demographics, these estimates show the total effect of the gaze dwell characteristic. In other words, if certain gaze patterns are correlated with demographics such as gender and age, these effects will be picking up that effect on risk preferences as well. We find that the percent of time spent dwelling on probabilities has a $-0.60(-1.09 \leftrightarrow-0.10)$ effect on $\varrho$, a $1.70(0.80 \leftrightarrow 2.60)$ effect on $\eta$, a $-0.06(-0.41 \leftrightarrow 0.28)$

${ }^{11}$ EUT and RDU (and CPT, for that matter) all agree on what the risk premium is for given choice data: they simply decompose it differently. Thus the fact that $\varrho<r$ implies that the net effect of the estimates of $\eta$ and $\varphi$, ceteris paribus $\varrho$, must be to generate a slight risk premium for this battery.

${ }^{12}$ As expected, this "inverse-S" shape generates pessimistic decision weights for 2-price lotteries, with the worst (best) outcome being overweighted (underweighted), resulting in a risk premium ceteris paribus $\varrho$. For 3-prize and 4-prize lotteries the characteristic over-weighting of extremes is evident, but with an asymmetry leading to greater over-weighting of the worst outcome, again generating a risk premium ceteris paribus $\varrho$.

${ }^{13}$ Estimates for an effect on the EUT parameter $r$ could not be found. In general, that parameter resisted efforts to be characterized as heterogeneous. 
effect on $\varphi$, a $0.04(-0.01 \leftrightarrow 0.09)$ effect on $\mu$, and a $0.43(-0.03 \leftrightarrow 0.90)$ effect on $\pi$. It has a statistically significant joint effect on the pwf parameters, as well as $\varrho, \eta, \varphi, \mu$ and $\pi$ as a whole.

Thus more time spent looking at probabilities is associated with greater decision-making using EUT $(\not$-value $=0.02)$, and much more pessimism generated by RDU probability weighting. This last effect can be seen in Figure 3. The average percent of time spent dwelling on probabilities is $40 \%$. If we reduce that percent from $40 \%$ to $20 \%$ we see choices consistent with the RDU specification in the top panels of Figure 3, with greater concavity of the utility function and less dramatic probability pessimism. If we increase that percent from $40 \%$ to $60 \%$ we see choices consistent with the RDU specification in the bottom panels of Figure 3, with convexity of the utility function and much more dramatic probability pessimism. Thus eye movements, as reflected in this one characteristic, help us identify significantly different types of RDU decision makers.

We can extend the analysis to study the marginal effect of eye movements, once we control for the effect of demographics. We consider covariates for gender, age, self-reported racial category "black," and self-reported high GPA between 3.75 and 4 for each structural parameter. The qualitative effect of greater percent dwell time on probabilities remains the same: much less concave utility functions for RDU decision makers, quickly becoming convex; much higher pwf parameter $\eta$ and no change to the pwf parameter $\varphi$, resulting in even greater probability pessimism than seen in the bottom panels of Figure 3; and no change in the probability of choices consistent with EUT $(\pi)$ or behavioral noise $(\mu)$. The effects on $\varrho$ and $\pi$ are statistically significant at the 0.07 level.

Finally, we can consider the effect of including a covariate to reflect the time spent on the decision. This is not something that one needs an eye-tracker for, and is a common characteristic collected automatically in software for experiments (e.g., Ztree). Adding this covariate by itself generates no statistically significant total effect on any individual structural parameter, and the joint effect on $\mathrm{r}, \varrho, \eta$, $\varphi, \mu$ and $\pi$ is insignificant with a $p$-value of 0.63 . Adding this covariate when demographics are included 
as well as the effect of the percent dwell time results in no statistically significant marginal effect on $\varrho, \eta$, $\varphi, \mu$ and $\pi$ considered by themselves, but it does jointly affect the pwf parameters $\eta$ and $\varphi$, and the parameters $\varrho, \eta, \varphi, \mu$ and $\pi$, in each case at a $p$-value of 0.1 . Adding duration reduces the statistical effect of percent dwell time on probabilities on $\varrho$, but does not change the significance of the effect on the pwf parameter $\eta$, discussed above in relation to Figure 3. Thus one cannot simply substitute response duration for the information from an eye-tracker.

\section{Implications and Extensions}

Our approach has an immediate implication for the normative design of choice displays for risky products. If we view certain features of risk preference as normatively unattractive, such as probability weighting in the presence of objective probabilities, then one can evaluate alternative designs that encourage decision making processes that mitigate it. For us, this is the over-riding reason for examining structural models, since they provide the basis for normative policy evaluation.

An immediate extension is to consider behavior at the level of the individual. This has become the standard manner in which we estimate risk preferences for descriptive and normative purposes (e.g., Harrison and Ng [2016] and Harrison and Ross [2018]). There are good reasons to think that studying individual behavior, rather than the behavior of representative agents, will make a major difference in the case of evaluations of the effects of decision-making processes, which can be a priori expected to exhibit considerable individual heterogeneity.

Another extension is to use eye-tracking to assess display screens that were not designed for eye-tracking. Many of the displays used for eye-tracking research, including ours in Figure 1, are deliberately designed to isolate areas of interest to enhance inferential power. But many of the displays in the field that communicate risk, such as warning labels, are not designed with eye-tracking in mind. To what extent do the lessons from these artefactual displays apply to natural or naturalistic displays? 
An immediate corollary of answers to these descriptive questions will be normative hypotheses that can then be tested with the latter displays.

Yet another extension is to use eye-tracking to investigate alternative elicitation procedures in the literature when the procedures yield apparently divergent results. ${ }^{14}$ By capturing eye-process data associated with alternative elicitation procedures, one could gain insight into aspects of the decision process leading to the different outcomes.

Another direction for eye-tracking research in decision making could be a focus on so-called choice anomalies. When analyzing only choices, patterns may emerge that lead an observer to conclude that there is evidence of systematic anomalous behavior. But when additional process data is added to the analysis, the once-seemingly-anomalous behavior may ultimately prove to be succinctly rationalized. For example, preference reversals have already been studied with an eye-tracker (Kim, Seligman and Kable [2012] and Rubaltelli, Dickert and Slovic [2012]). Perhaps similar investigations can shed new light on other choice paradoxes, including the Ellsberg paradox, Allais paradox, gambler's fallacy, and other puzzles.

One application of eye-tracking might be to generate better statistical calibration functions to correct for hypothetical bias in decisions under risk. The evidence for hypothetical bias is clear if one reads the full literature (see Holt and Laury [2005] and Harrison [2006]). Is there some identifiable pattern in the processing of attributes of lotteries in hypothetical settings that differs from the pattern in incentivized settings? If so, one might be able to correct the former to better reflect the latter. It is easy to say, "stop being cheap, and just run the experiment with incentives and avoid hypothetical bias," and we will continue to say just that in laboratory settings. But in many instances this is

${ }^{14}$ Recent examples with respect to the elicitation of risk preferences include Crosetto and Filippin [2016] and Zhou and Hey [2018]. Unlike many comparisons of elicitation methods, these studies consider the implications for inferred risk preferences, which is what matters, and do not worry about naive correlations of observed choice patterns across elicitation methods. 
inefficient, such as when hypothetical questions can be cheaply asked of vast samples, but incentivizied experiments can only be conducted on smaller sub-samples.

We have used only dwell times and barely scratched the surface of the rich process data obtained from an eye-tracker. None of the sequence dynamics of the process data are used, and there are several ways that one could imagine this being done. One is to look at scan paths to try to identify lexicographic patterns, such as the "editing phase" and "evaluation phase" proposed by Kahneman and Tversky [1979] and neglected in subsequent literature on CPT. 


\section{Figure 1: Eye-Tracking Choice Display}

\begin{tabular}{|ll|}
\hline$\$ 57$ & $40 \%$ \\
\hline$\$ 9$ & $45 \%$ \\
\hline$\$ 36$ & $5 \%$ \\
\hline$\$ 12$ & $10 \%$ \\
\hline
\end{tabular}

\begin{tabular}{|ll|}
\hline$\$ 27$ & $90 \%$ \\
\hline$\$ 74$ & $10 \%$ \\
\hline
\end{tabular}

Border included here to convey the screen edges and relative positioning of display elements on a full screen. Actual display did not include this border. 
Figure 2: Probability Weighting and Decision Weights Estimated Prior to Adding Eye-Tracker Data
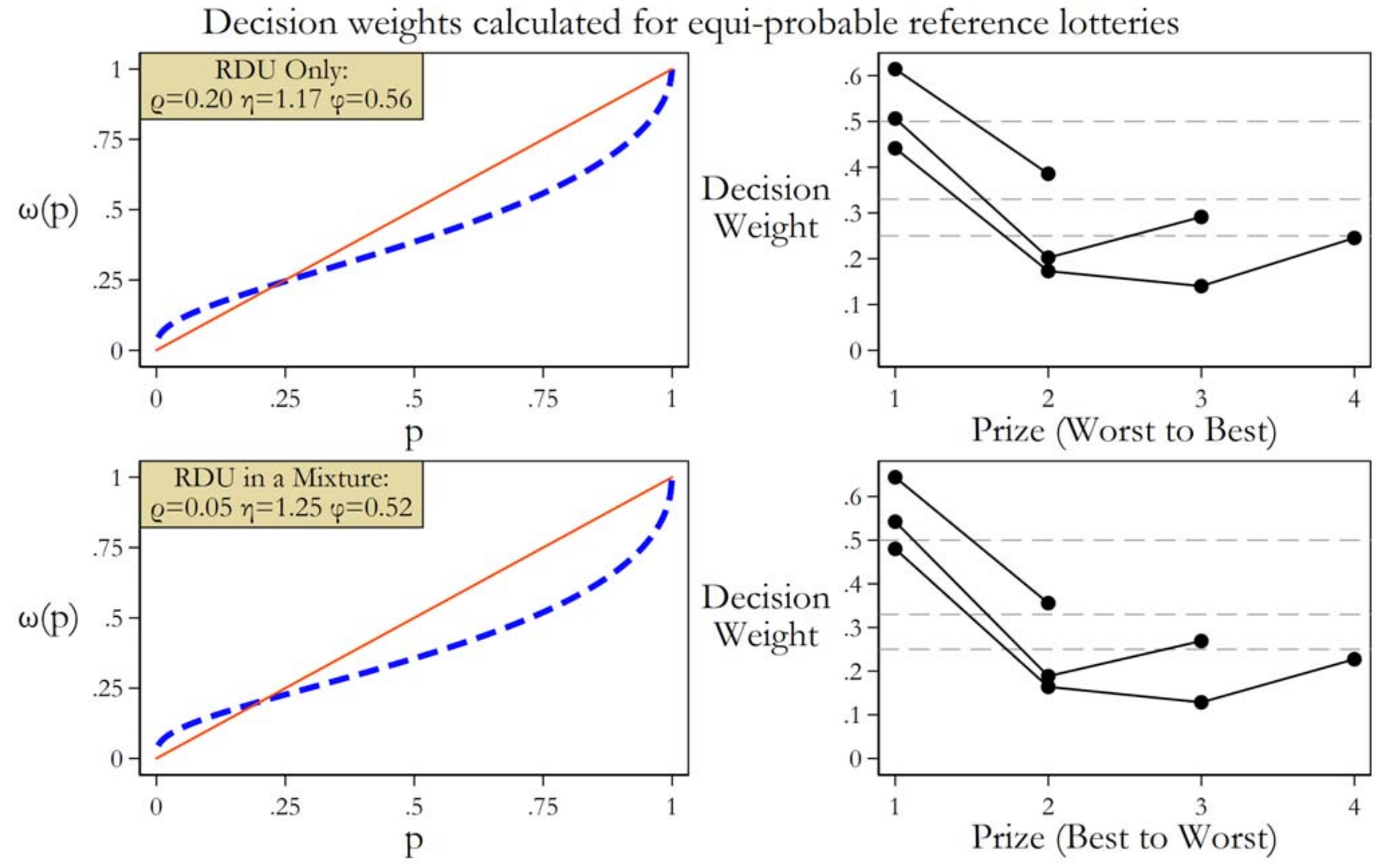


\section{Figure 3: Effect of Changes in Probability Dwell Proportions on Probability Weighting and Decision Weights}

Decision weights calculated for equi-probable reference lotteries
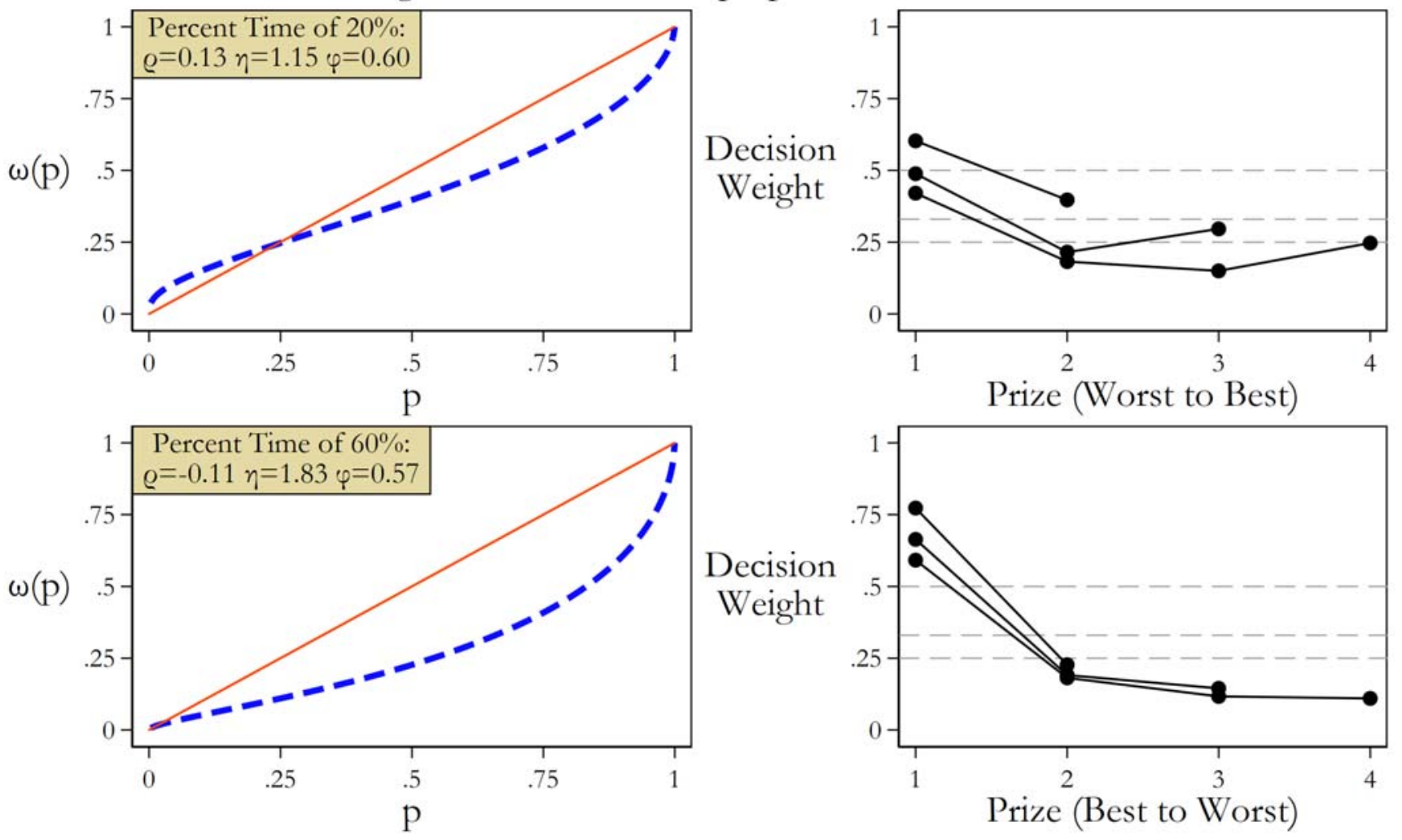


\section{References}

Arieli, Amos; Ben-Ami, Yaniv, and Rubinstein, Ariel, "Tracking Decision Makers under Uncertainty," American Economic Journal: Microeconomics, 3(4), November 2011, 68-76.

Böckenholt, Ulf, and Hynan, Linda S., "Caveats on a Process-Tracing Measure and a Remedy," Journal of Behavioral Decision Making, 7, 1994a, 103-117.

Böckenholt, Ulf, and Hynan, Linda S., "Similarities and Differences Between SI and SM: A Reply to Payne and Bettman," Journal of Behavioral Decision Making, 7, 1994b, 123-127.

Crosetto, Paolo, and Filippin, "A Theoretical and Experimental Appraisal of Four Risk Elicitation Methods,” Experimental Economics, 19, 2016, 613-641.

Fiedler, Susann, and Glöckner, Andreas, "The Dynamics of Decision Making in Risky Choice: An EyeTracking Analysis," Frontiers in Psychology, 3, October 2012, Article 335, 1-18.

Glöckner, Andreas; Fiedler, Susann; Hochman, Guy; Ayal, Shahar, and Hilbig, Benjamin E., "Processing Differences Between Descriptions and Experience: A Comparative Analysis Using Eye-Tracking and Physiological Measures," Frontiers in Psychology, 3, June 2012, Article 173, 1-15.

Glöckner, Andreas, and Herbold, Ann-Katrin, “An Eye-Tracking Study on Information Processing in Risky Decisions: Evidence for Compensatory Strategies Based on Automatic Processes," Journal of Behavioral Decision Making, 24, 2011, 71-98.

Harrison, Glenn W., “Hypothetical Bias Over Uncertain Outcomes,” in J.A. List (ed)., Using Experimental Methods in Environmental and Resource Economics (Northampton, MA: Elgar, 2006).

Harrison, Glenn W., "Neuroeconomics: A Critical Reconsideration,” Economics \& Philosophy, 24(3), 2008, 303-344.

Harrison, Glenn W.; Hofmeyr, Andre; Ross, Don, and Swarthout, J. Todd, "Risk Preferences, Time Preferences, and Smoking Behavior," Southern Economic Journal, 85(2), 2018, 313-348.

Harrison, Glenn W., and Ng, Jia Min, "Evaluating the Expected Welfare Gain of Insurance," Journal of Risk \& Insurance, 83(1), March 2016, 91-120.

Harrison, Glenn W., and Ross, Don, "Varieties of Paternalism and the Heterogeneity of Utility Structures," Journal of Economic Methodology, 25(1), 2018, $42-67$.

Harrison, Glenn W., and Rutström, E. Elisabet, "Risk Aversion in the Laboratory," in J.C. Cox and G.W. Harrison (eds.), Risk Aversion in Experiments (Bingley, UK: Emerald, Research in Experimental Economics, Volume 12, 2008).

Harrison, Glenn W., and Rutström, E. Elisabet, "Expected Utility And Prospect Theory: One Wedding and a Decent Funeral," Experimental Economics, 12(2), June 2009, 133-158. 
Holt, Charles A., and Laury, Susan K., "Risk Aversion and Incentive Effects: New Data Without Order Effects," American Economic Review, 95(3), June 2005, 902-912.

Janowski, Vanessa, Computational Biases in Decision-Making, Ph.D Thesis Dissertation, California Institute of Technology, 2012.

Just, Marcel Adam, and Carpenter, Patricia A., "A Theory of Reading: From Eye Fixations to Comprehension,” Psychological Review, 87(4), July 1980, 329-354.

Kahneman, Daniel, and Tversky, Amos, "Prospect Theory: An Analysis of Decision Under Risk," Econometrica, 47, 1979, 263-291.

Kim, Betty E.; Seligman, Darryl, and Kable, Joseph W., "Preference Reversal in Decision Making Under Risk Are Accompanied by Changes in Attention to Different Attributes," Frontiers in Neuroscience, 6, July 2012, Article 109, 1-10.

Lohse, Gerald L., and Johnson, Eric J., "A Comparison of Two Process Tracing Methods for Choice Tasks," Organizational Behavior and Human Decision Processes, 68(1), October 1996, 28-43.

Orquin, Jacob L., and Loose, Simone Mueller, "Attention and Choice: A Review on Eye Movements in Decision Making," Acta Psychologica, 144, 2013, 190-206.

Posner, Michael, “Orienting of Attention,” Quarterly Journal of Experimental Psychology, 32, 1980, 3-25.

Prelec, Drazen, “The Probability Weighting Function,” Econometrica, 66, 1998, 497-527.

Quiggin, John, “A Theory of Anticipated Utility,” Journal of Economic Behavior \& Organization, 3(4), 1982, 323-343.

Rayner, Keith, "Eye Movements in Reading and Information Processing: 20 Years of Research," Psychological Bulletin, 124(3), 1998, 372-422.

Rayner, Keith, "Eye Movements and Attention in Reading, Scene Perception, and Visual Search," Quarterly Journal of Experimental Psychology, 62(8), 2009, 1457-1506.

Rosen, Larry D., and Rosenkoetter, Paul, "An Eye Fixation Analysis of Choice and Judgment with Multiattribute Stimuli,” Memory \& Cognition, 4(6), 1976, 747-752.

Rubaltelli, Enrico; Dickert, Stehpan, and Slovic, Paul, "Response Mode, Compatibility, and DualProcesses in the Evaluation of Simple Gambles: An Eye-Tracking Investigation," Judgment and Decision Making, 7(4), July 2012, 427-440.

Russo, J. Edward, and Dosher, Barbara Anne, "Strategies for Multiattribute Binary Choice," Journal of Experimental Psychology: Learning, Memory and Cognition, 9, 1983, 676-696.

Su, Yin; Rao, Li-Lin; Sun, Hong-Yue; Du, Xue-Lei; Li, Xingshan, and Li, Shu, "Is Making a Risky Choice Based on a Weighting and Adding Process? An Eye-Tracking Investigation," Journal of Experimental Psychology: Learning, Memory and Cognition, 39(6), 2013, 1765-1780. 
Stewart, Neil; Hermens, Frouke, and Matthews, William J., "Eye Movements in Risky Choice," Journal of Behavioral Decision Making, 29, 2016, 116-136.

Zhou, Wenting, and Hey, John, “Context Matters,” Experimental Economics, 21, 2018, 723-756. 


\section{Appendix A: Instructions and Parameters (Online Working Paper)}

Word versions of these instructions are available in an archive at

\section{https://cear.gsu.edu/gwh/}

with a link that matches the title of this paper.

These instructions were presented in the order shown here.

Videos for each instruction were presented to subjects, to ensure that session-specific effects were minimized. The archive at the above link includes these MP4 files. The longer, main video provided images of the dice used to generate random numbers, displayed in the video as that text was read out aloud (in the video) from the instructions. 


\section{A. Introductory Text}

\section{Eye Tracking}

To better understand how you make your decisions in this experiment, we will record your eye movements with an eye-tracking device. This device is essentially a camera underneath your computer screen that will tell us where you are looking on the screen at any moment. The camera is recording only information about your eye movements, and stores this information as numbers. The camera never records any image of you.

After we finish the experiment instructions, we will spend a few minutes adjusting the eye-tracking system to best record your eye movements. You will be asked to look at a series of circles on your screen so that we can focus the system to your eyes. We may also have to reposition your chair or make other minor adjustments to better configure the system.

Please let the experimenter know if you wear contact lenses, and whether they are hard or soft lenses. Sometimes we must adjust the system to account for contact lenses.

The eye-tracker can track your eyes if you wear glasses, but certain styles of glasses may create reflections which interfere with the sysem. In case you have glasses and we see reflections from them, we will first try to adjust the system to eliminate the reflections. But if the adjustments do not work, we may need to place a piece of tape on your glasses to block the reflections. Alternatively, you may instead remove your glasses if you can read the screen without glasses. 


\section{Choices Over Risky Prospects}

In today's experiment you will choose between prospects with varying prizes and chances of winning. You will be presented several pairs of prospects, and for each pair you will choose the prospect you prefer. You will make choices over a number of pairs. You will actually play one of the prospects you choose, and you will be paid according to the outcome of that prospect, so you should think carefully about which prospect you prefer.

\section{Making Choices}

Here is an example of what the computer display of a pair of prospects will look like.
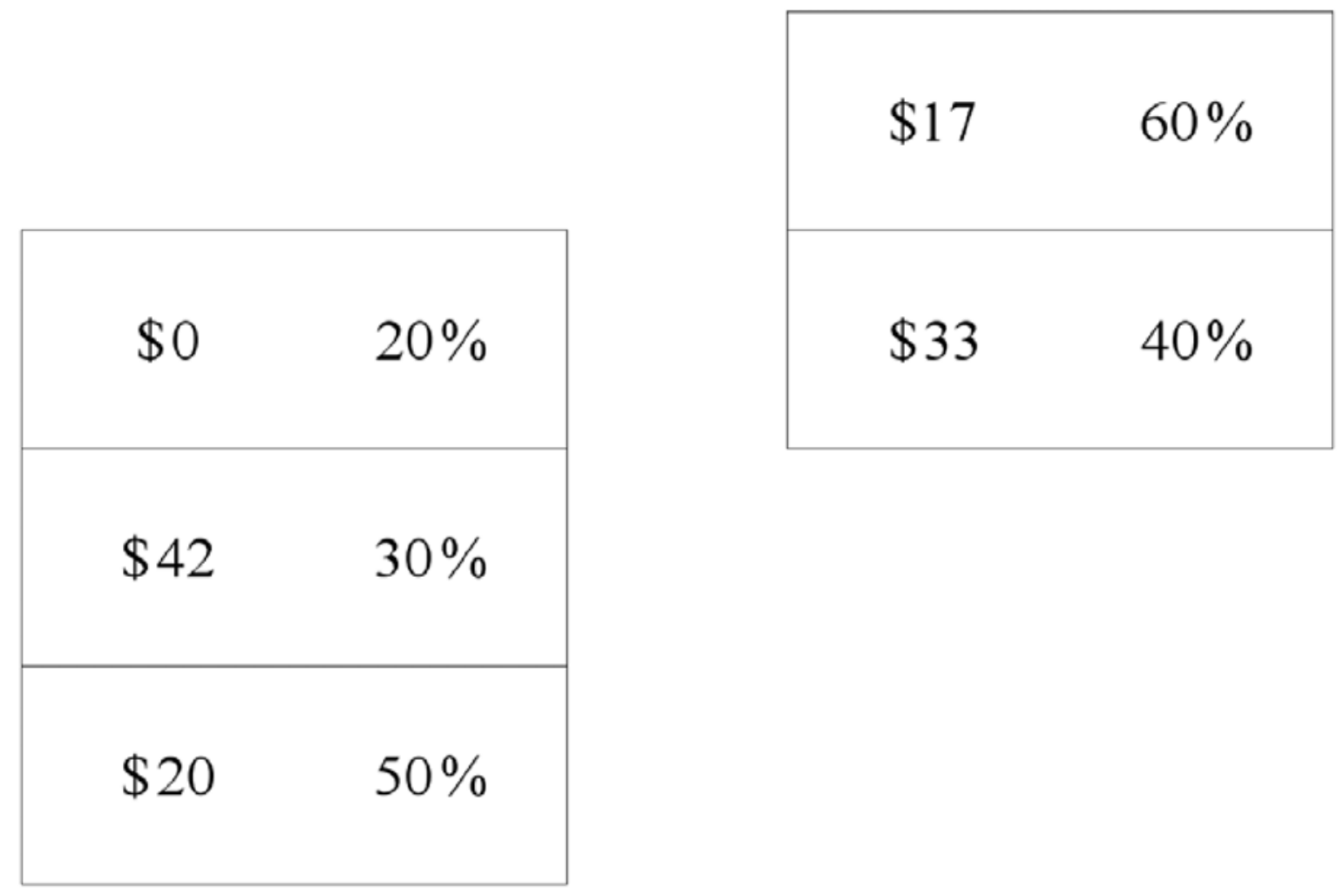

In this example, we see the left prospect has a $20 \%$ chance paying $\$ 0$, a $30 \%$ chance of paying $\$ 42$, and a $50 \%$ chance of paying $\$ 20$. Looking now at the right prospect, we see it has a $60 \%$ chance of paying $\$ 17$, and a $40 \%$ chance of paying $\$ 33$.

You will select your preferred prospect by pressing on one of the two buttons on the button box in front of you. For example, if you prefer the left prospect then you would press the left button. Similarly, if you prefer the right prospect then you would press the right button. This works best if you place both hands on the button box, and then use your left hand for the left button and your right hand for the right button. 
Since there is a chance that any of your choices may be played for real cash, you should approach each decision as if it is the one that you will play out.

Before each choice screen, a target will be displayed on the monitor. You must look at the target in order to move on to the choice screen. If you want to pause during the experiment, please do so on a target screen before looking at the target. This will halt the software from displaying your next choice.

\section{Playing a prospect and getting paid}

After you have worked through all the pairs of prospects, you will then play one of your selected prospects.

First, you will roll two 10-sided dice until a number comes up to determine which of your choices will be played. For example, if you had made 20 choices, you would roll until a number between 1 and 20 comes up. If instead you had made 50 choices, you would roll until a number between 1 and 50 comes up. And so on.

The experimenter will then display on your screen the corresponding choice you made. For example, if you rolled a 34, then the experimenter will display the 34th pair of prospects you saw, along with your choice. Here is an example of how the screen will look when you play a choice for cash.
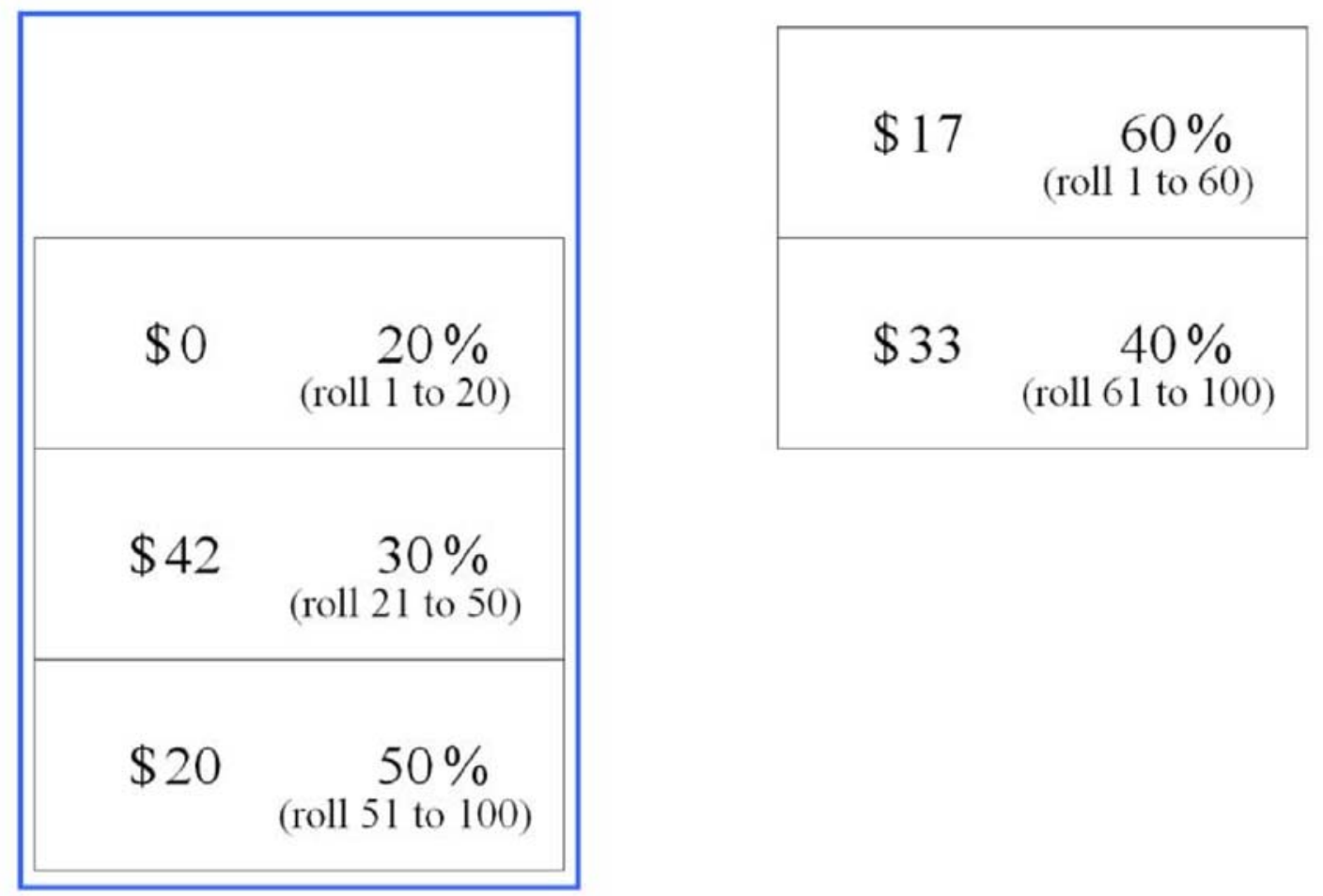

Notice the blue box around the left prospect. This blue box shows that you selected the left prospect during the decision phase of the experiment. If you had selected the right prospect instead, then the blue box would have instead appeared around the right prospect. You can not change your 
choice at this point in the experiment.

Next you will roll the two 10-sided dice again to determine the payment you receive from the prospect you chose. Notice that the screen now displays how this roll will determine the possible payment amounts. For example, looking at the selected left prospect above, if you roll a 9, then you would be paid $\$ 0$. If instead you rolled a 37 , then you would be paid $\$ 42$. And if instead you rolled a 73 , then you would be paid $\$ 20$.

\section{Summary}

1. Which prospects you prefer is a matter of personal taste. Please work silently, and make your choices by thinking carefully about each prospect.

2. You will select your preferred prospect in each pair by pressing the left or right button on the button box.

3. If you want to pause while making decisions, please do so on a target screen before looking at the target.

4. Your payoff in this experiment is determined by three things:

1. by which prospect you select, the left or the right, for each of the pairs;

2. by which prospect pair is chosen to be played out when you roll the two 10-sided dice the first time; and

3. by the outcome of your chosen prospect when you roll the two 10-sided dice the second time.

5. All payoffs are in cash, and are in addition to the $\$ 5$ show-up payment that you receive just for being here. 


\section{Lotteries for the Standard Risk. Aversion Task with No Eye-Tracking}

The lottery parameters are listed in Table A1. Column qid refers to the ID for each question. The columns starting with the text prob refer to probabilities, and the columns starting with the text prize refer to monetary prizes. After the "prob" or "prize" text is a number, 1, 2, 3 and 4, that refers to the $1^{\text {st }}, 2^{\text {nd }}, 3^{\text {rd }}$ and $4^{\text {th }}$ outcome in each lottery. Finally, after these numbers the letter $\mathbf{L}$ denotes the Left lottery in the display and the letter $\mathbf{R}$ denotes the Right lottery in the display. 
Table A1: Parameters for the Risk Aversion Lottery Battery

See text for explanation of lottery names and variables

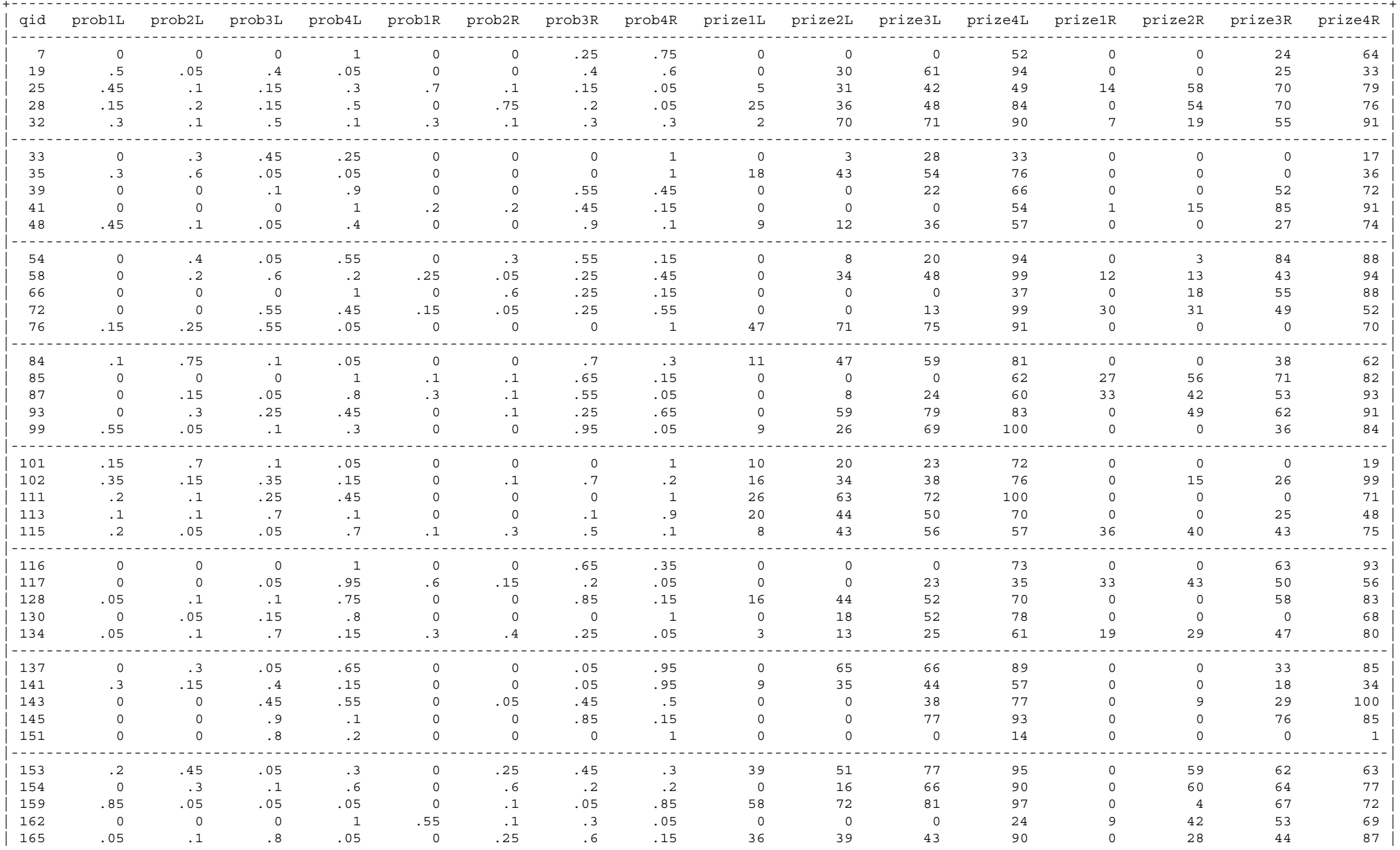




\begin{tabular}{|c|c|c|c|c|c|c|c|c|c|c|c|c|c|c|c|c|}
\hline 170 & 4 & .3 & .25 & .05 & 0 & .35 & .25 & .4 & 10 & 84 & 87 & 99 & 0 & 38 & 48 & 70 \\
\hline 171 & 0 & .4 & .15 & .45 & .25 & .05 & .35 & .35 & 0 & 34 & 60 & 95 & 26 & 35 & 84 & 85 \\
\hline 173 & 0 & 0 & .8 & .2 & .4 & .25 & .2 & .15 & 0 & 0 & 27 & 94 & 19 & 33 & 50 & 92 \\
\hline 177 & 0 & 0 & .35 & .65 & 0 & .2 & .25 & .55 & 0 & 0 & 32 & 54 & 0 & 16 & 17 & 74 \\
\hline 178 & 0 & 0 & .4 & .6 & 0 & .5 & .05 & .45 & 0 & 0 & 47 & 53 & 0 & 23 & 42 & 83 \\
\hline 181 & .2 & .1 & .05 & .65 & 0 & .55 & .4 & .05 & 9 & 31 & 53 & 86 & 0 & 43 & 77 & 83 \\
\hline 185 & .05 & .5 & .15 & .3 & 0 & 0 & 0 & 1 & 1 & 8 & 22 & 65 & 0 & 0 & 0 & 23 \\
\hline 187 & 0 & .35 & .1 & .55 & .6 & .05 & .15 & .2 & 0 & 9 & 46 & 65 & 24 & 35 & 70 & 94 \\
\hline 194 & 0 & 0 & 0 & 1 & 0 & 0 & .4 & .6 & 0 & 0 & 0 & 70 & 0 & 0 & 60 & 78 \\
\hline 197 & 0 & .05 & .1 & .85 & 0 & .35 & .5 & .15 & 0 & 4 & 32 & 67 & 0 & 37 & 58 & 92 \\
\hline
\end{tabular}




\section{Appendix B: Estimating Structural Models of Decision-Making (Online Working Paper)}

We write out the formal econometric specifications for EUT and RDU models, to be applied to determine the probability that individual subjects behave consistently with EUT and RDU in a mixture model. The exposition here repeat certain equations from the main text so as to be selfcontained.

\section{A. Expected Utility}

Assume that utility of income is defined by

$$
\mathrm{U}(\mathrm{x})=\mathrm{x}^{(1-\mathrm{r})} /(1-\mathrm{r})
$$

where $\mathrm{x}$ is the lottery prize and $\mathrm{r} \neq 1$ is a parameter to be estimated. For $\mathrm{r}=1$ assume $\mathrm{U}(\mathrm{x})=\ln (\mathrm{x})$ if needed. Thus $\mathrm{s}$ is the coefficient of CRRA: $r=0$ corresponds to risk neutrality, $r<0$ to risk loving, and $\mathrm{r}>0$ to risk aversion. Let there be J possible outcomes in a lottery. Under EUT the probabilities for each outcome $\mathrm{x}_{\mathrm{j}}, \mathrm{p}\left(\mathrm{x}_{\mathrm{j}}\right)$, are those that are induced by the experimenter, so expected utility is simply the probability weighted utility of each outcome in each lottery i:

$$
\mathrm{EU}_{\mathrm{i}}=\sum_{\mathrm{j}=1, \mathrm{~J}}\left[\mathrm{p}\left(\mathrm{x}_{\mathrm{j}}\right) \times \mathrm{U}\left(\mathrm{x}_{\mathrm{j}}\right)\right] .
$$

The EU for each lottery pair is calculated for a candidate estimate of $r$, and the index

$$
\nabla \mathrm{EU}=\mathrm{EU}_{\mathrm{R}}-\mathrm{EU}_{\mathrm{L}}
$$

calculated, where $\mathrm{EU}_{\mathrm{L}}$ is the "left" lottery and $\mathrm{EU}_{\mathrm{R}}$ is the "right" lottery as presented to subjects. This latent index, based on latent preferences, is then linked to observed choices using a standard cumulative normal distribution function $\Phi(\nabla \mathrm{EU})$. This "probit" function takes any argument between $\pm \infty$ and transforms it into a number between 0 and 1 . Thus we have the probit link function,

$$
\operatorname{prob}(\text { choose lottery } \mathrm{R})=\Phi(\nabla \mathrm{EU})
$$

Even though this "link function" is common in econometrics texts, it is worth noting explicitly and understanding. It forms the critical statistical link between observed binary choices, the latent structure generating the index $\nabla \mathrm{EU}$, and the probability of that index being observed. The index defined by (B3) is linked to the observed choices by specifying that the R lottery is chosen when $\Phi(\nabla E U)>1 / 2$, which is implied by (B4).

Thus the likelihood of the observed responses, conditional on the EUT and CRRA specifications being true, depends on the estimates of $r$ given the above statistical specification and the observed choices. The "statistical specification" here includes assuming some functional form for the cumulative density function (CDF). The conditional log-likelihood is then

$$
\ln \mathrm{L}(\mathrm{r} ; \mathrm{y}, \mathbf{X})=\sum_{\mathrm{i}}\left[\left(\ln \Phi(\nabla \mathrm{EU}) \times \mathbf{I}\left(\mathrm{y}_{\mathrm{i}}=1\right)\right)+\left(\ln (1-\Phi(\nabla \mathrm{EU})) \times \mathbf{I}\left(\mathrm{y}_{\mathrm{i}}=-1\right)\right)\right]
$$

where $\mathbf{I}(\cdot)$ is the indicator function, $\mathrm{y}_{\mathrm{i}}=1(-1)$ denotes the choice of the right (left) lottery in risk aversion task $\mathrm{i}$, and $\mathbf{X}$ is a vector of individual characteristics reflecting age, sex, race, and so on. 
Harrison and Rutström [2008; Appendix F] review procedures that can be used to estimate structural models of this kind, as well as more complex non-EUT models. The goal is to illustrate how researches can write explicit maximum likelihood (ML) routines that are specific to different structural choice models. It is a simple matter to correct for multiple responses from the same subject ("clustering"), as needed for the pooled estimation results we present.

An important extension of the core model is to allow for subjects to make some behavioral errors. The notion of error is one that has already been encountered in the form of the statistical assumption that the probability of choosing a lottery is not 1 when the EU of that lottery exceeds the EU of the other lottery. This assumption is clear in the use of a non-degenerate link function between the latent index $\nabla \mathrm{EU}$ and the probability of picking one or other lottery; in the case of the normal $\mathrm{CDF}$, this link function is $\Phi(\nabla \mathrm{EU})$. If there were no errors from the perspective of EUT, this function would be a step function: zero for all values of $\nabla \mathrm{EU}<0$, anywhere between 0 and 1 for $\nabla E U=0$, and 1 for all values of $\nabla E U>0$.

We employ the error specification originally due to Fechner and popularized by Hey and Orme [1994]. This error specification posits the latent index

$$
\nabla \mathrm{EU}=\left(\mathrm{EU}_{\mathrm{R}}-\mathrm{EU}_{\mathrm{L}}\right) / \mu
$$

instead of (B3), where $\mu$ is a structural "noise parameter" used to allow some errors from the perspective of the deterministic EUT model. This is just one of several different types of error story that could be used, and Wilcox [2008] provides an excellent review of the implications of the alternatives. As $\mu \rightarrow 0$ this specification collapses to the deterministic choice EUT model, where the choice is strictly determined by the EU of the two lotteries; but as $\mu$ gets larger and larger the choice essentially becomes random. When $\mu=1$ this specification collapses to (B3), where the probability of picking one lottery is given by the ratio of the EU of one lottery to the sum of the EU of both lotteries. Thus $\mu$ can be viewed as a parameter that flattens out the link functions as it gets larger.

An important contribution to the characterization of behavioral errors is the "contextual error" specification proposed by Wilcox [2011]. It is designed to allow robust inferences about the primitive "more stochastically risk averse than," and posits the latent index

$$
\nabla \mathrm{EU}=\left[\left(\mathrm{EU}_{\mathrm{R}}-\mathrm{EU}_{\mathrm{L}}\right) / \nu\right] / \mu
$$

instead of $\left(\mathrm{B}^{\prime}\right)$, where $v$ is a new, normalizing term for each lottery pair $\mathrm{L}$ and $\mathrm{R}$. The normalizing term $\nu$ is defined as the maximum utility over all prizes in this lottery pair minus the minimum utility over all prizes in this lottery pair. The value of $\nu$ varies, in principle, from lottery choice pair to lottery choice pair: hence it is said to be "contextual." For the Fechner specification, dividing by $v$ ensures that the normalized $\mathrm{EU}$ difference $\left[\left(\mathrm{EU}_{\mathrm{R}}-\mathrm{EU}_{\mathrm{I}}\right) / \nu\right]$ remains in the unit interval. The term $\nu$ does not need to be estimated in addition to the utility function parameters and the parameter for the behavioral error tern, since it is given by the data and the assumed values of those estimated parameters.

The specification employed here is the CRRA utility function from (B1), the Fechner error specification using contextual utility from $\left(\mathrm{B}^{\prime \prime}\right)$, and the link function using the normal CDF from (B4). The log-likelihood is then 


$$
\ln \mathrm{L}(\mathrm{r}, \mu ; \mathrm{y}, \mathbf{X})=\sum_{\mathrm{i}}\left[\left(\ln \Phi(\nabla \mathrm{EU}) \times \mathbf{I}\left(\mathrm{y}_{\mathrm{i}}=1\right)\right)+\left(\ln (1-\Phi(\nabla \mathrm{EU})) \times \mathbf{I}\left(\mathrm{y}_{\mathrm{i}}=-1\right)\right)\right]
$$

and the parameters to be estimated are s and $\mu$ given observed data on the binary choices $y$ and the lottery parameters in $\mathbf{X}$. The matrix $\mathbf{X}$ can also contain information on demographic characteristics of the subjects, as well as characteristics of the task.

It is possible to consider more flexible utility functions than the CRRA specification in (1), but that is not essential for present purposes.

\section{B. Rank-Dependent Utility}

The RDU model of Quiggin [1982] extends the EUT model by allowing for decision weights on lottery outcomes. The specification of the utility function is the same parametric specification (B1) considered for EUT, but with $\mathrm{r}$ replaced with $\varrho$. To calculate decision weights $\mathrm{w}(\cdot)$ under RDU one replaces expected utility defined by (B3) with RDU

$$
\mathrm{RDU}_{\mathrm{i}}=\sum_{\mathrm{j}=1, \mathrm{~J}}\left[\mathrm{w}\left(\mathrm{p}\left(\mathrm{x}_{\mathrm{j}}\right)\right) \times \mathrm{U}\left(\mathrm{x}_{\mathrm{j}}\right)\right]=\sum_{\mathrm{j}=1, \mathrm{~J}}\left[\mathrm{w}_{\mathrm{j}} \times \mathrm{U}\left(\mathrm{x}_{\mathrm{j}}\right)\right]
$$

where

$$
\mathrm{w}_{\mathrm{j}}=\omega\left(\mathrm{p}_{\mathrm{j}}+\ldots+\mathrm{p}_{\mathrm{J}}\right)-\omega\left(\mathrm{p}_{\mathrm{j}+1}+\ldots+\mathrm{p}_{\mathrm{J}}\right)
$$

for $j=1, \ldots, J-1$, and

$$
\mathrm{w}_{\mathrm{j}}=\omega\left(\mathrm{p}_{\mathrm{j}}\right)
$$

for $\mathrm{j}=\mathrm{J}$, with the subscript $\mathrm{j}$ ranking outcomes from worst to best, and $\omega(\cdot)$ is some probability weighting function.

We use a probability weighting function proposed by Prelec [1998] that exhibits considerable flexibility. This function is

$$
\omega(\mathrm{p})=\exp \left\{-\eta(-\ln \mathrm{p})^{\varphi}\right\}
$$

and is defined for $0<p \leq 1, \eta>0$ and $\varphi>1$. When $\varphi=1$ this function collapses to the Power function $\omega(\mathrm{p})=\mathrm{p}^{\eta}$.

The construction of the log-likelihood for the RDU model the Prelec probability weighting requires the estimation of the parameters $\varrho, \eta, \varphi$ and $\mu$.

\section{Mixture Models}

It is possible to extend this analysis by thinking of the observed choices as a mixture of two distinct latent data-generating processes, rather than one data-generating process (EUT) or the other (RDU). If we let $\pi^{\mathrm{EUT}}$ denote the probability that the EUT process is correct, and $\pi^{\mathrm{RDU}}=\left(1-\pi^{\mathrm{EUT}}\right)$ denote the probability that the RDU process is correct, the grand likelihood of the EUT/RDU 
process as a whole can be written as the probability weighted average of the conditional likeliboods. If we define the likelihoods for the $i^{\text {th }}$ observation under the EUT (RDU) model by $l_{i}^{\text {EUT }}\left(l_{i}^{\text {RDU }}\right)$, then the grand likelihood for the overall EUT/RDU mixture model is

$$
\ln \mathrm{L}\left(\mathrm{r}, \varrho, \eta, \varphi \mu, \pi^{\mathrm{EUT}} ; \mathrm{y}, \mathbf{X}\right)=\sum_{\mathrm{i}} \ln \left[\left(\pi^{\mathrm{EUT}} \times l_{\mathrm{i}}^{\mathrm{EUT}}\right)+\left(\pi^{\mathrm{RDU}} \times l_{\mathrm{i}}^{\mathrm{RDU}}\right)\right] \text {. }
$$

This log-likelihood can be maximized to find estimates of the parameters of each latent process, as well as the mixing probability $\pi^{\mathrm{EUT}}$. The probability estimate is constrained to lie in the unit interval by estimating a parameter $\zeta$ and defining $\pi^{\mathrm{EUT}}=1 /(1+\exp (\zeta))$ inside the likelihood function. The literal interpretation of the mixing probabilities is at the level of the observation.

This approach assumes that any one observation can be generated by both models, although it admits of extremes in which one or other criterion wholly generates the observation. One could alternatively define a grand likelihood in which observations or subjects are classified as following one model or the other on the basis of the latent probabilities $\pi^{\mathrm{EUT}}$ and $\pi^{\mathrm{RDU}}$. El-Gamal and Grether [1995] illustrate this approach in the context of identifying behavioral strategies in Bayesian updating experiments. However, in the case of the EUT and RDU models, it is natural to view the tension between the models as reflecting different instances of the lottery choice problem: for example, 2prize lotteries might be evaluated using EUT, but for 3-prize of 4-prize lotteries RDU might be used. Thus we do not believe it would be consistent with the EUT and RDU models to categorize choices as wholly driven either by EUT or RDU.

These priors also imply that we prefer not to use mixture specifications in which subjects are categorized as completely EUT or RDU. It is possible to rewrite the grand likelihood (B8) such that $\pi$ ${ }_{\mathrm{i}}^{\text {EUT }}=1$ and $\pi_{\mathrm{i}}^{\mathrm{RDU}}=0$ if $l_{\mathrm{i}}^{\text {EUT }}>l_{\mathrm{i}}^{\mathrm{RDU}}$, and $\pi_{\mathrm{i}}^{\mathrm{EUT}}=0$ and $\pi_{\mathrm{i}}^{\mathrm{RDU}}=1$ if $l_{\mathrm{i}}^{\text {EUT }}<l_{\mathrm{i}}^{\mathrm{RDU}}$, where the subscript i now refers to the individual subject. The general problem with this specification is that it assumes that there is no effect on the probability of EUT and RDU from task domain. We do not want to impose that assumption, even for a relatively homogenous task design such as ours.

\section{Additional References}

El-Gamal, Mahmoud A., and Grether, David M., “Are People Bayesian? Uncovering Behavioral Strategies," Journal of the American Statistical Association, 90, 432, December 1995, 1137-1145.

Hey, John D., and Orme, Chris, "Investigating Generalizations of Expected Utility Theory Using Experimental Data," Econometrica, 62(6), November 1994, 1291-1326.

Wilcox, Nathaniel T., "Stochastic Models for Binary Discrete Choice Under Risk: A Critical Primer and Econometric Comparison," in J. Cox and G.W. Harrison (eds.), Risk. Aversion in Experiments (Bingley, UK: Emerald, Research in Experimental Economics, Volume 12, 2008).

Wilcox, Nathaniel T., “'Stochastically More Risk Averse:' A Contextual Theory of Stochastic Discrete Choice Under Risk," Journal of Econometrics, 162(1), May 2011, 89-104. 


\section{Appendix C: Previous Literature (Online Working Paper)}

Rosen and Roisenkoetter [1976] appears to be the first eye-tracking study of choice over risky lotteries. Their motivation was to determine if choice over risky lotteries was "holistic," in the sense that the EU of each lottery is evaluated, and then the choice made on the basis of which is larger. The alternative is a "dimensional" pattern in which the utility of one lottery is compared to the utility of the other lottery one dimension at a time, and then some additive function used to evaluate which lottery to choose. In the case of risky lotteries, one of their three types of stimuli, one dimension is prizes and the other dimension is probabilities. Evaluating by dominance relations is the most common dimensional approach. The always had three attributes in each lottery: a positive payoff, a probability for that positive payoff, and a negative payoff. The probability for the negative payoff was implied as 1 minus the probability of the positive payoff. Their lottery pairs always made the dimensions interdependent, in the sense that some tradeoff was needed. ${ }^{15}$ Six subjects were paid $\$ 1.88$ an hour to participate, so incentives were not salient with respect to choices. Transitions between fixations were classified as dimensional, holistic, or other. Focusing just on the first two, $38 \%$ of the transitions were dimensional and $62 \%$ holistic (p. 750 ). Of course, the gamble design had been set up to favor holistic processing.

Russo and Dosher [1983] extended this design to allow for gambles that favored dimensional processing as well as gambles that favored holistic processing. Each lottery had two outcomes, with one outcome always a zero payoff with the residual probability. Thus the display consisted of four numbers: a probability and non-zero payoff for one lottery, and a probability and non-zero payoff for the other lottery. Over 60 choices, in half the cases the "winning attribute" was probability (payoffs),

\footnotetext{
${ }^{15}$ One example is lottery A, with prizes $+\$ 4.29$ and $-\$ 1.29$, and probability for the positive prize of 0.44 , compared to lottery $\mathrm{B}$, with prizes +2.85 and $-\$ 2.80$, and probability for the positive prize of 0.72 . So a dimensional subject might see that B favors $A$ with respect to the positive prize size, but A favors B with respect to the probability on that prize. So "knowledge about the probability cannot easily be evaluated in the absence of information about the corresponding payoffs," (p. 748) encouraging a holistic processing strategy.
} 
in the sense that the other attribute was held constant across the two lotteries and one probability (payoffs) varied. Subjects were paid to participate, but rewards were not salient even though non-zero payoffs were only between $\$ 2.60$ and $\$ 4.60$. Subjects were first asked to choose their preferred lottery in each instance, and then asked to select the lottery with the highest EV in each instance, for 120 choices in total. Out of 10 subjects, 4 exhibited primarily holistic processing, 4 exhibited primary dimensional processing, 1 exhibited both, and 1 was essentially random. ${ }^{16}$

Arieli, Ben-Ami and Rubinstein [2011] pursue the same strategy, to detect if subjects follow holistic strategies or what they call "component" procedures (which are the same as dimensional procedures in the prior literature). The display consisted of one lottery on the left with a positive payoff shown on top and the corresponding probability shown underneath, and another lottery on the right with a positive payoff on top and the corresponding probability underneath. In each case, zero was the other payoff with the implied probability. The posit that subjects that exhibit vertical eye transitions exhibit holistic processing, and subjects that exhibit horizontal eye transitions exhibit component or dimensional processing. Subjects were paid $\$ 12$ to participate, with no salient rewards. ${ }^{17}$ Transitions were the basis for determining the type of eye movement. In two sets of problems in which the EV was relatively easy to compute, a slight majority of patterns favored holistic processing for 70 subjects, and in two sets of problems in which the EV was relatively hard to compute, a slight majority of patterns favored component or dimensional processing. But in all four

\footnotetext{
${ }^{16}$ Based on a minimum number of 3 fixations for each IA, subjects were allocated to holistic transitions, dimensional transitions, and unclassified transitions. The highest fraction of the first two was used to determine the type of decision-making process. For instance, subject \#9 (Table 5, p.690) had 2,738 fixations, of which $37 \%$ led to dimensional transitions, $21 \%$ to holistic transitions, and $43 \%$ were unclassified; this subject was classified overall as dimensional. Most subjects classified as dimensional or holistic had a much higher fraction allocated to that type of transition.

${ }^{17}$ Arieli et al. [2011; p.69] claim that there "is ample evidence that the lack of monetary incentives does not significantly affect participants' choices," despite clear evidence to the contrary surveyed by Harrison [2006].
} 
sets of problems the fraction of both types of processing was high (Table 1, p.72). ${ }^{18}$

Glöckner and Herbold [2011] consider the same general issue, but motivated by different theories of decisions under risk. They view EUT and Cumulative Prospect Theory (CPT) as both proposing holistic strategies, ${ }^{19}$ and contrast this with the Priority Heuristic $(\mathrm{PH})$ due to Brandstätter, Gigerenzer and Hertwig [2006], which is indeed dimensional and lexicographic. ${ }^{20}$ Two additional process models from psychology are considered. In fact, since they restrict their lotteries to the gain domain, it is not CPT that they are considering but RDU. Their hypotheses for each theory are stated (p.77) in vague, qualitative terms. For example, one hypothesis for CPT (RDU) is that decision time should be the same for each task, and another hypothesis is that the amount of inspected information is the same for all tasks. Of course, one could imagine one subject with a sharply "inverse-S" pwf, who would effectively just be inspecting the information on the highest ranked prize and the lowest ranked prize, in contrast with someone that has a barely concave or convex "power" pwf who would care more or less equally about all prizes. Thus these hypotheses bear no relation to the variations within CPT (RDU), unless one constrains them arbitrarily. ${ }^{21}$ Each of 18 subjects completed 40 binary choice tasks, for a fixed, non-salient payoff of $€ 18$. At least in terms of the comparison of CPT (RDU) and PH, the results, based on fixations and transitions, clearly support the former.

Fiedler and Glöckner [2012] is important because it appears to be the first eye-tracking study

\footnotetext{
${ }^{18}$ For the two easy sets, it was $24 \%, 23 \%, 18 \%$ and $28 \%$ and then $20 \%, 25 \%, 25 \%$ and $23 \%$, where the first two percentages are vertical transitions and the last two percentages are horizontal transitions. For the two harder sets, it was $17 \%, 18 \%, 20 \%$ and $30 \%$ and then $16 \%, 18 \%, 33 \%$ and $28 \%$.

${ }^{19}$ Prospect theory in general is actually a mix of presumed processing strategies. If one goes back to the original Prospect Theory of Kahneman and Tversky [1979], there were two processing stages presumed to be applied in sequence. One was an "editing" stage which applied dominance principles, among other heuristics, to simplify the task. This stage is clearly dimensional. If the editing stage did not lead to a clear dominance-based choice, the subject then engaged in a holistic "evaluation" phase. Sadly, the CPT of Tversky and Kahneman [1992] seems to have edited away the editing stage.

${ }^{20}$ The PH has some serious limitations in it's ability to account for the most basic of patterns in choice under risk: see Andersen, Harrison, Lau and Rutström [2010; §7].

${ }^{21}$ This is what is done by Glöckner and Herbold [2011; p.74], who take the estimates from Tversky and Kahneman [1992] as if they apply precisely for every subject.
} 
that provided salient rewards to lottery choice. ${ }^{22}$ Subjects received a show-up fee of $€ 6$ as well as the outcome of playing out one of the selected choices from a battery of 50 choices. Average payoffs were low, by our standards: $€ 6.20$ in Study 1 and $€ 9.20$ in Study 2. However, the range of payoffs was quite wide: between $€ 0$ and just over $€ 49$ in each study. They extend the design of Glöckner and Herbold [2011] by varying the average EV and difference in EV across lottery pairs. Their analysis was agnostic about specific models of choice under risk, but focused on the dynamics of choice and how it varied with probability, payoff value, and their interaction. They regress the number of fixations on each of these covariates over all subjects (21 and 37 in Study1 and Study 2, respectively), allowing for random effects to capture unobserved heterogeneity of individuals. These results (Table 4, p.7) show that "attention to an outcome of a gamble increases with its probability and its value and that attention shifts towards the subsequently favored gamble after about two-thirds of the decision process" (p.1).

Janowski [2012; chapter II] is important because it adopts a structural approach to understanding if eye movements can explain the levels of loss aversion that subjects exhibit in their choices. An explicit, structural CPT model, of sorts, is proposed and estimated for each subject. The model assumes away any probability weighting, and assumes that the CRRA for the intrinsic utility function is the same for losses as it is for gains. ${ }^{23}$ Subjects face an interface that shows one gain prize and probability (e.g., $+\$ 10$ with probability 0.2 ) and one loss prize and probability (e.g., $-\$ 5$ with probability 0.3$)$. The implied probability $(0.5)$ is applied to payoff of $\$ 0$. The choice between this mixed-frame lottery was also implied: the alternative lottery was $\$ 0$ for certain. There is no mention of an endowment to cover losses, so presumably this was paid out of the "show-up fee and

${ }^{22}$ Along with a closely related study by Glöckner, Fiedler, Hochman, Ayal and Hilbig [2012]. Their focus is the extent to which eye-tracking and skin-conductance measures provide more information to allow one to differentiate the cognitive processes when probabilities are "described" (i.e., shown on the interface, as in our experiments) or "experienced" (i.e., learned over time from sample realizations).

${ }^{23}$ In the notation of Tversky and Kahneman [1992], it is assumed that $\alpha=\beta$. 
experiment completion fee" (p. 72). Subjects were incentivized by being paid for 5 out of a staggering 384 choices, raising concerns with portfolio effects on choice. ${ }^{24}$ The main results draw on correlations between the point estimate of the loss aversion parameter $\lambda$ for each of 20 subjects and the percentage of time looking at the gain prize minus the percentage of time looking at the loss prize, presumably over all 384 choices. Hence these are correlations of 20 numbers with 20 numbers, which is quite a small sample. This correlation also makes no statistical sense: the point estimate of a parameter is not data, it is a random variable. Hence the finding of a positive correlation, while intuitive enough, cannot be taken seriously, quite apart from doubts about whether these estimates capture loss aversion correctly since probability weighting was assumed away.

Su et al. [2013] also used salient rewards: 49 subjects received a show-up fee of $¥ 60 \mathrm{RMB}$, average salient payoffs were $¥ 28 \mathrm{RMB}$, and the range of payoffs was between $¥ 0 \mathrm{RMB}$ and $¥ 45$ RMB. Each subject made 32 choices over risky lotteries, in which there were two non-negative prizes and both probabilities were displayed. The primary hypothesis was whether cognitive processes would be different if subjects faced one realization of the lottery of choice in a pair, or faced the EV (over 100 realizations) of the lottery of choice in a pair. The latter treatment would presumably encourage holistic or "compensatory" processing, particularly since there were no dominated choices. Another treatment was to have half of their lottery pairs use computationally easy, rounded prizes and probabilities, and the other half use computationally harder prizes and probabilities. One aspect of their analysis was to compare choice predictions against the predictions of specific models, including risk-neutrality, EUT and CPT (RDU). Unfortunately the predictions for the latter two models used specific, arbitrary point estimates for structural coefficients that do not reflect the

\footnotetext{
24 The notion of "choice" is itself unusual. Subjects were asked to indicate if the "strongly accepted the gamble," "weakly accepted the gamble," "weakly rejected the gamble," or "strongly rejected the gamble." Presumably the first two choices implied acceptance, and the last two choices implied rejection.
} 
generality of the model. ${ }^{25} \mathrm{~A}$ more interesting finding is that the fraction of transitions that are holistic rather than dimensional is much higher when the payoff metric is $\mathrm{EV}$, whether or not the lottery pair values are computationally easy or hard. ${ }^{26}$

Stewart, Hermens and Matthews [2016] used "barely salient" rewards: subjects received $\AA^{3}$ for participating, and a salient reward between $£^{0}$ and $£^{2.50}$. The lottery choice prizes ranged

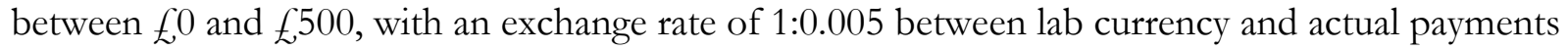
(remarkably, revealed to subjects at the end of the experiment). Rounded lottery prizes and probabilities were selected to be computationally easy. The interface displayed one prize and probability for each lottery, with a $f^{0}$ prize receiving the implied residual probability. The battery consisted of 75 choices, with 4 of these involving stochastically dominated alternatives. The remaining choices had a median EV difference of $£ 150$, and were designed to capture a variety of "risky" and "safe" choices for various presumed levels of risk aversion. A deliberately a-theoretical analysis is adopted, using statistical models to descriptively characterize eye movements. They start by looking at fixations on attributes, and show that there is approximate balance between prizes and probabilities, irrespective of the size of each. They then focus on eye movement patterns and choice, and conclude that the simple accumulation of dwell time on a lottery better predicts the eventual choice than the patterns of dwell time. This latter result is consistent with one of the key findings of Fiedler and Glöckner [2012], that "attention shifts towards the subsequently favored gamble after about two-thirds of the decision process" (p.1).

\section{Additional References}

\footnotetext{
${ }^{25}$ For EUT a log utility function is assumed, and for CPT (RDU) the "point estimates" from Tversky and Kahneman [1992] are assumed.

26 The summary statistic used in this instance is the "search measure" SM index proposed by Böckenholt and Hynan [1994a], and discussed by Payne and Bettman [1994] and Böckenholt and Hynan [1994b].
} 
Andersen, Steffen; Harrison, Glenn W.; Lau, Morten, and Rutström, Elisabet, "Behavioral Econometrics for Psychologists," Journal of Economic Psychology, 31, 2010, 553-576.

Brandstätter, Eduard; Gigerenzer, Gerd, and Hertwig, Ralph, “The Priority Heuristic: Making Choices Without Trade-offs," Psychological Review, 113, 2006, 409-432.

Böckenholt, Ulf, and Hynan, Linda S., "Caveats on a Process-Tracing Measure and a Remedy," Journal of Behavioral Decision Making, 7, 1994a, 103-117.

Böckenholt, Ulf, and Hynan, Linda S., "Similarities and Differences Between SI and SM: A Reply to Payne and Bettman," Journal of Behavioral Decision Making, 7, 1994b, 123-127.

Fiedler, Susann, and Glöckner, Andreas, "The Dynamics of Decision Making in Risky Choice: An Eye-Tracking Analysis," Frontiers in Psychology, 3, October 2012, Article 335, 1-18.

Glöckner, Andreas; Fiedler, Susann; Hochman, Guy; Ayal, Shahar, and Hilbig, Benjamin E., "Processing Differences Between Descriptions and Experience: A Comparative Analysis Using Eye-Tracking and Physiological Measures," Frontiers in Psychology, 3, June 2012, Article $173,1-15$.

Glöckner, Andreas, and Herbold, Ann-Katrin, "An Eye-Tracking Study on Information Processing in Risky Decisions: Evidence for Compensatory Strategies Based on Automatic Processes," Journal of Behavioral Decision Making, 24, 2011, 71-98.

Janowski, Vanessa, Computational Biases in Decision-Making, Ph.D Thesis Dissertation, California Institute of Technology, 2012.

Payne, John W., and Bettman, James R., "The Costs and Benefits of Alternative Measures of Search Behavior: Comments on Böckenholt and Hynan," Journal of Behavioral Decision Making, 7, 1994, 119-122.

Rosen, Larry D., and Rosenkoetter, Paul, "An Eye Fixation Analysis of Choice and Judgment with Multiattribute Stimuli," Memory \& Cognition, 4(6), 1976, 747-752.

Russo, J. Edward, and Dosher, Barbara Anne, "Strategies for Multiattribute Binary Choice," Journal of Experimental Psychology: Learning, Memory and Cognition, 9, 1983, 676-696.

Su, Yin; Rao, Li-Lin; Sun, Hong-Yue; Du, Xue-Lei; Li, Xingshan, and Li, Shu, "Is Making a Risky Choice Based on a Weighting and Adding Process? An Eye-Tracking Investigation," Journal of Experimental Psychology: Learning, Memory and Cognition, 39(6), 2013, 1765-1780.

Stewart, Neil; Hermens, Frouke, and Matthews, William J., "Eye Movements in Risky Choice," Journal of Behavioral Decision Making, 29, 2016, 116-136.

Tversky, Amos, and Kahneman, Daniel, "Advances in Prospect Theory: Cumulative Representations of Uncertainty," Journal of Risk \& Uncertainty, 5, 1992, 297-323. 


\section{Appendix D: Detailed Estimates (Online Working Paper)}

Estimates are reported for each of the models referred to in the text. Figures 2 and 3 are generated by Stata command files figure2.do and figure3.do, respectively, and require no data. All other estimates are generated by Stata command file Main.do. The data compilation code is included to document the procedures used, but the estimation data is provided to allow that stage to be skipped (this also ensures confidentiality of individual subjects). Data and code for replication is available in an archive at https://cear.gsu.edu/gwh/, with a link that matches the title of this paper.

\section{Estimates of EUT Model with No Covariates}

- ml model lf ML_eut ( $r$ : choiceR \$Rdata =) (mu:), cluster(sid) maximize difficult $\operatorname{init}(.51$, copy)

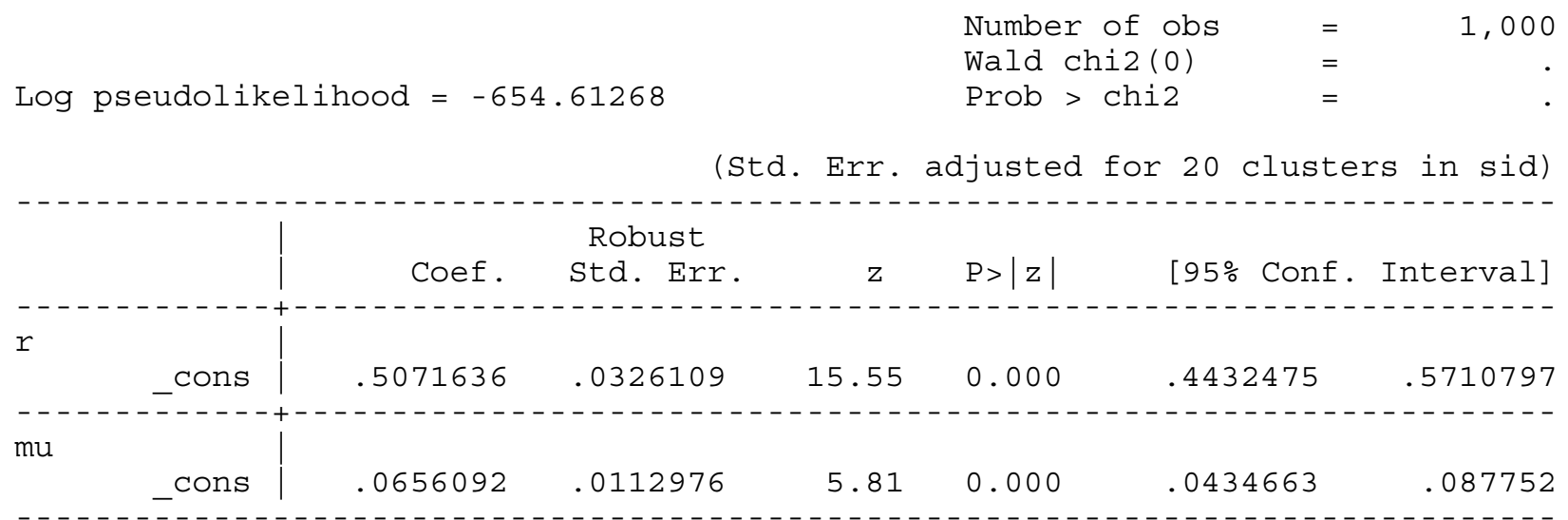

\section{Estimates of RDU Model with No Covariates}

- ml model lf ML_rdu_prelec2c ( $r$ : choiceR \$Rdata = ) (LNeta:) (LNphi:) (mu: ), cluster(sid) maximizē difficult technique(bfgs) init('rEUT' 0.024 -1.89 'muEUT', copy)

Log pseudolikelihood $=-587.52147$
(Std. Err. adjusted for 20 clusters in sid)

$\begin{array}{llr}\text { Number of obs } & = & 1,000 \\ \text { Wald chi2 }(0) & = & . \\ \text { Prob > chi2 } & = & .\end{array}$


mu cons $\quad \begin{array}{rrrrrr}0798412 & .015685 & 5.09 & 0.000 & .0490992 & .1105832\end{array}$

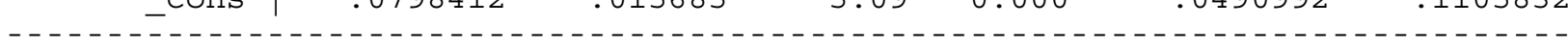

- $n l$ com (eta: $\exp ([$ LNeta]_b[_cons $]))(\operatorname{phi}: \exp ([$ LNphi]_b[_cons $]))$

eta: $\exp ([$ LNeta]_b [_cons] $)$

phi : exp ([LNphi]_b[_cons $])$

\begin{tabular}{|c|c|c|c|c|c|c|}
\hline & Coef. & std. Err. & $\mathrm{z}$ & $\mathrm{P}>|\mathrm{z}|$ & {$[95 \%$ Conf } & Interval] \\
\hline & 7 & --- & 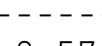 & ----- & 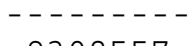 & 1701010 \\
\hline $\begin{array}{l}\text { eta } \\
\text { phi }\end{array}$ & $\begin{array}{l}1.170675 \\
.5600719\end{array}$ & $\begin{array}{l}.1223592 \\
.0663992\end{array}$ & $\begin{array}{l}9.57 \\
8.43\end{array}$ & $\begin{array}{l}0.000 \\
0.000\end{array}$ & $\begin{array}{l}.9308557 \\
.4299317\end{array}$ & $\begin{array}{r}1.410495 \\
.690212\end{array}$ \\
\hline
\end{tabular}

* test EUT

- testnl $(\exp ([$ LNeta]_b[_Cons $])=1)(\exp ([$ LNphi]_b[_cons $])=1)$, mtest (b)

(1) $\exp ([$ LNeta $]$ b [_cons $])=1$

(2) $\exp ([$ LNphi]_b [_cons $])=1$

\begin{tabular}{|c|c|c|c|}
\hline & chi2 & $d f$ & $\mathrm{p}$ \\
\hline \multicolumn{4}{|c|}{ - - - - - + - - - - - - - - - - - - - - - - - - - - - - - - - - - - } \\
\hline (1) & 1.95 & 1 & $0.3261 \#$ \\
\hline (2) & 43.90 & 1 & 0.0000 \\
\hline \multicolumn{4}{|c|}{ - - - - - - + - - - - - - - - - - - - - - - - - - - - - - - - - } \\
\hline all & 54.06 & 2 & 0.0000 \\
\hline
\end{tabular}

\# Bonferroni-adjusted p-values

\section{Estimates of the EUT-RDU Mixture Model with No Covariates}

* * mixture of EUT and RDU, with Prelec pwf

- ml model lf ML_eut_rdu_prelec2c (rEUT: choiceR \$Rdata = ) (rRDU: ) (LNeta: ) (LNphi: ) (kappa: ) (mu: ) if qid_record==1, cluster(sid) maximize technique(dfp) difficult init('r' 'rPR' 'LNeta' 'LNphi' o 'mu_mix', copy)

Log pseudolikelihood $=-578.39289$

$\begin{array}{llr}\text { Number of obs } & = & 1,000 \\ \text { Wald chi2 }(0) & = & . \\ \text { Prob }>\text { chi2 } & = & .\end{array}$

(std. Err. adjusted for 20 clusters in sid)

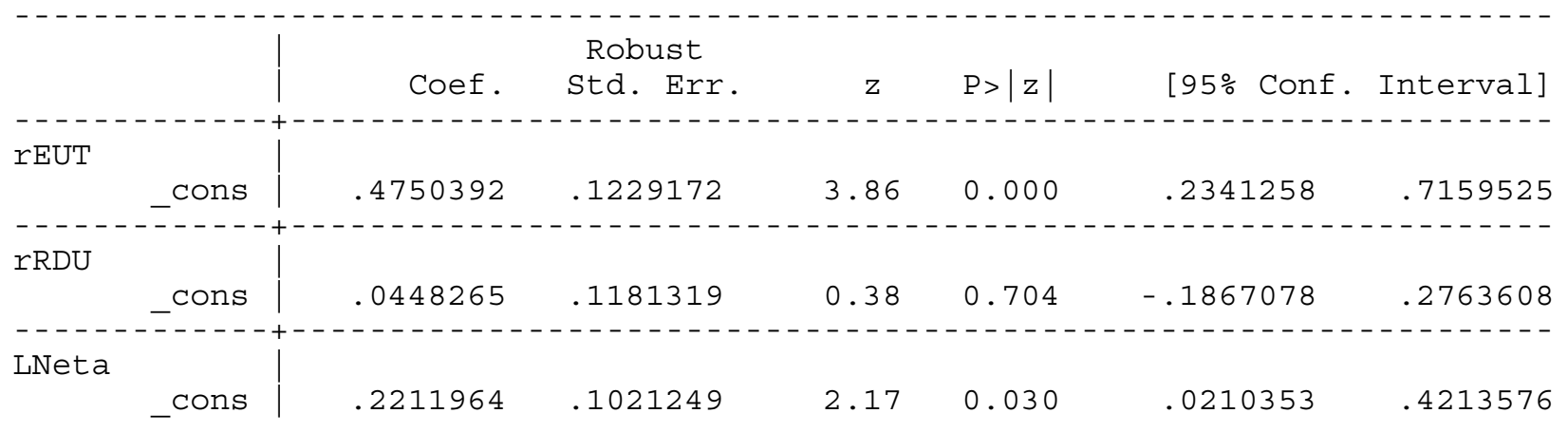




LNphi
kappa

- nlcom (eta: $\exp ([$ LNeta]_b [_cons $]))$ (phi : exp ([LNphi]_b [_cons])) (probEUT: $\left.1 /\left(1+\exp \left([\mathrm{kappa}]_{C} \mathrm{cons}\right)\right)\right)$ (prōbRDU: $1-(1 /(1+\exp ([\mathrm{kapp} \bar{a}]$ cons $\left.)))\right)$

eta: $\exp ([$ LNeta]_b [_cons $])$

phi : $\exp ([$ LNphi] $\mathrm{b}[$ Cons $])$

probEUT: $1 /(1+\exp ([\mathrm{k} \bar{a} p \bar{p} \bar{a}]$ cons $))$

probRDU: $\quad 1-(1 /(1+\exp ([\mathrm{k}$ appa $]$ _cons $)))$

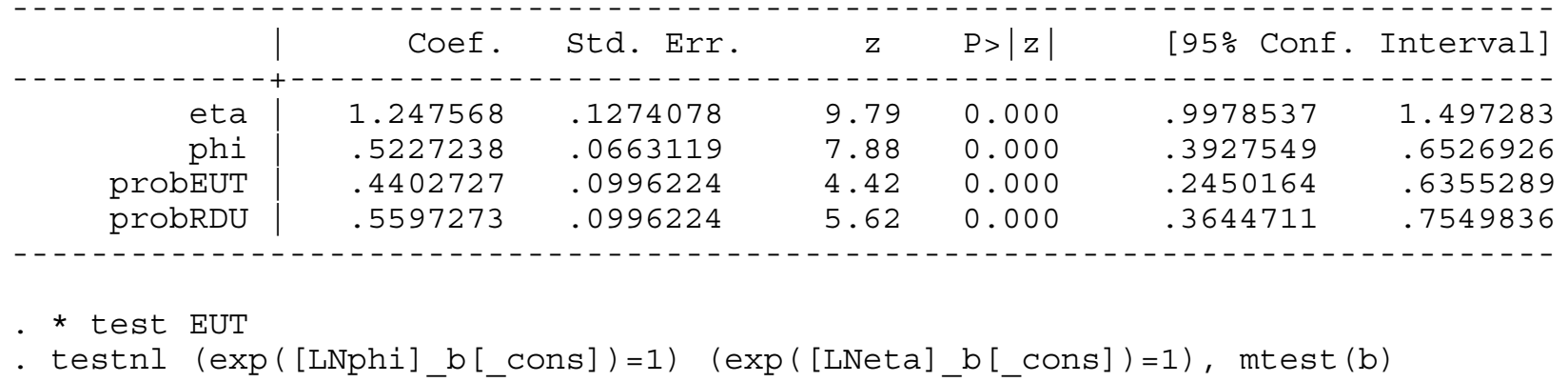

(1) $\exp ([$ LNphi]_b [_cons $])=1$

(2) $\exp ([$ LNeta $]$ b [_cons $])=1$

\begin{tabular}{|c|c|c|c|}
\hline & chi2 & $\partial \mathrm{f}$ & $\mathrm{p}$ \\
\hline (1) & 51.80 & 1 & $0.0000 \quad \#$ \\
\hline (2) & 3.78 & 1 & $0.1040 \#$ \\
\hline---- & ----- & -- & -------- \\
\hline all & 86.69 & 2 & 0.0000 \\
\hline
\end{tabular}

\# Bonferroni-adjusted p-values

\section{Estimates of the EUT-RDU Mixture Model with Eye-Tracking Covariates Only}

- ml model lf ML_eut_rdu_prelec2c (rEUT: choiceR \$Rdata = ) (rRDU: \$eyes ) (LNeta: \$eyes ) (LNphi: \$eyes ) (kâppa: \$eyes ) (mu: \$eyes) if qid_record==1, cluster(sid) maximize technique(nr) difficult continue

Log pseudolikelihood $=-558.90315$

$\begin{array}{llr}\text { Number of obs } & = & 1,000 \\ \text { Wald chi2 }(0) & = & \text {. } \\ \text { Prob }>\text { chi2 } & = & \end{array}$

(std. Err. adjusted for 20 clusters in sid)

Robust
Coef. Std. Err. $\quad$ z $\quad$ P $>|z| \quad$ [95\% Conf. Interval]




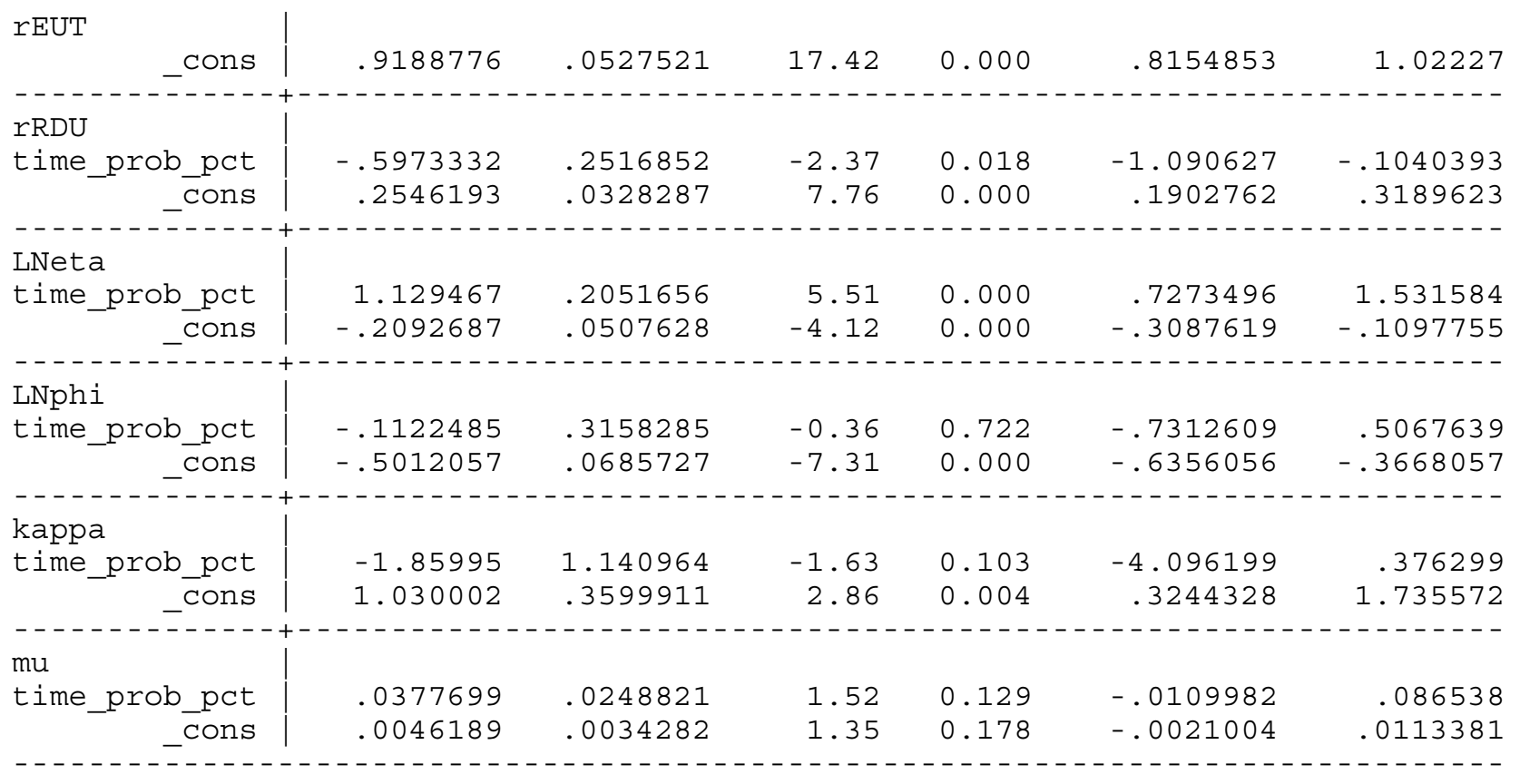

- nlcom (rEUT: [rEUT]_cons) (rRDU: [rRDU]_cons) (eta: exp ([LNeta]_b [_cons])) (phi :

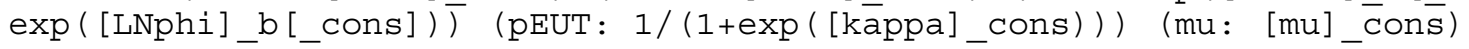

\begin{tabular}{|c|c|}
\hline rEUT : & [rEUT]_cons \\
\hline rRDU : & {$[r \mathrm{RDU}]$ _cons } \\
\hline eta: & $\exp ([$ LN $e t a]$ b [_cons $])$ \\
\hline phi: & $\exp ([$ LNphi] $]$ b [_cons $])$ \\
\hline $\begin{aligned} \mathrm{pEUT} & \text { : } \\
\mathrm{mu} & \text {. }\end{aligned}$ & $\begin{array}{l}1 /(1+\exp ([\mathrm{k} \overline{a p p} \bar{a}] \text { cons })) \\
{[\mathrm{mu}] \text { cons }}\end{array}$ \\
\hline
\end{tabular}

\begin{tabular}{|c|c|c|c|c|c|c|}
\hline & Coef. & Std. Err. & $z$ & $\mathrm{P}>|\mathrm{z}|$ & [95\% Conf. & Interval] \\
\hline & & & 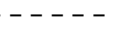 & & 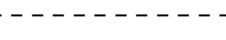 & 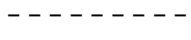 \\
\hline rEUT & .9188776 & .0527521 & 17.42 & 0.000 & .8154853 & 1.02227 \\
\hline rRDU & .2546193 & .0328287 & 7.76 & 0.000 & .1902762 & 3189623 \\
\hline eta & .8111772 & .0411776 & 19.70 & 0.000 & .7304706 & .8918838 \\
\hline phi & .6057998 & .0415413 & 14.58 & 0.000 & .5243804 & .6872193 \\
\hline pEUT & .2630836 & .0697917 & 3.77 & 0.000 & .1262944 & .3998729 \\
\hline $\mathrm{mu}$ & .0046189 & .0034282 & 1.35 & 0.178 & -.0021004 & .0113381 \\
\hline
\end{tabular}

rRDU_time_ t: [rRDU]_cons+[rRDU] time_prob_pct - [rRDU]_cons eta_Eime_p $\sim t: \exp ([\mathrm{L} \overline{\mathrm{N}} e t a]$ cons+[LNetä]time_prob_pct) - $\exp$ ([LNeta]_cons) phi_time_p t: $\exp ([\mathrm{LNphi}]$ Cons+[LNphi]time_prob_pct) - exp ([LNphi]_cons)

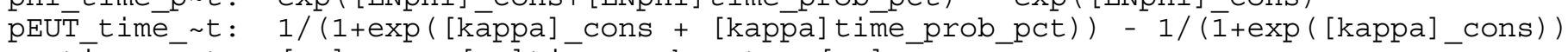
mu_time_prot: [mu]_cons+[mu]time_prob_pct - [mu]_cons

\begin{tabular}{|c|c|c|c|c|c|c|}
\hline & Coef & Std. Err. & $\mathrm{z}$ & $\mathrm{P}>|\mathrm{z}|$ & {$[95 \% \mathrm{Co}$} & Interval \\
\hline & & - & -- & & & \\
\hline rRDU_time_prob_pct & -.5973332 & .2516852 & -2.37 & 0.018 & -1.090627 & -.1040393 \\
\hline eta_time_prob_pct & 1.69861 & .4575269 & 3.71 & 0.000 & .801874 & 2.595347 \\
\hline phi_time_prob_pct & -.0643225 & .1747552 & -0.37 & 0.713 & -.4068364 & .2781913 \\
\hline pEUT_time_prob_pct & .4332602 & .2365893 & 1.83 & 0.067 & -.0304463 & .8969667 \\
\hline mu_time_prob_pct & .0377699 & .0248821 & 1.52 & 0.129 & -.0109982 & .086538 \\
\hline
\end{tabular}


( 1$)$ [rRDU]time_prob_pct $=0$

$\begin{aligned} \operatorname{chi2}(1)= & 5.63 \\ \text { Prob }>\text { chi2 } & =0.0176\end{aligned}$

( 1$)$ [LNeta] time_prob_pct $=0$

( 2) [LNphi]time_prob_pct $=0$

$\operatorname{chi2}(2)=37.35$

Prob $>$ chi2 $=0.0000$

( 1) [rRDU] time_prob_pct $=0$

( 2) [LNeta] time_prob_pct $=0$

( 3 ) [LNphi] time_prob_pct $=0$

( 4) [kappa]time_prob_pct $=0$

( 5) [mu]time_prob_pct $=0$

$\begin{aligned} \text { Chi2 }(5)= & 89.57 \\ \text { Prob }>\text { chi2 } & 0.0000\end{aligned}$

Estimates of the EUT-RDU Mixture Model with Duration Covariates Only

- ml model lf ML_eut_rdu_prelec2c (rEUT: choiceR \$Rdata = ) (rRDU: duration ) (LNeta: duration ) (LNphīi dūration ) (kappa: duration ) (mu: duration ) if qid_record==1, cluster(sid) maximize technique(nr) difficult continue

$\begin{array}{lll} & \text { Number of obs } & =1,000 \\ \text { Log pseudolikelihood }=-574.75976 & = & \text { Wald chi2(0) } \\ \text { Prob }>\text { chi2 } & =\end{array}$.

(Std. Err. adjusted for 20 clusters in sid)

\begin{tabular}{|c|c|c|c|c|c|c|}
\hline & \multirow{2}{*}{\multicolumn{6}{|c|}{\begin{tabular}{cccr|r} 
Coef. & Std. Err. & Z & P $>|z|$ & [95\% Conf. Interval $]$
\end{tabular}}} \\
\hline & & & & & & \\
\hline _cons & .469195 & .1205272 & 3.89 & 0.000 & .232966 & .7054241 \\
\hline$----\overline{-}----$ & ------ & --------- & . - & ---- & ------- & -- \\
\hline \multicolumn{7}{|l|}{ rRDU } \\
\hline duration & .0083107 & .0095044 & 0.87 & 0.382 & -.0103175 & .026939 \\
\hline _cons & .0162007 & .122688 & 0.13 & 0.895 & -.2242634 & .2566648 \\
\hline$-------\overline{-}----$ & --- & --------- & ---- & ----- & ----------- & --- \\
\hline \multicolumn{7}{|l|}{ LNeta } \\
\hline duration & .0209031 & .017838 & 1.17 & 0.241 & -.0140588 & .055865 \\
\hline _cons & .0792039 & .1538802 & 0.51 & 0.607 & -.2223957 & .38 \\
\hline--- & -- & ----- & ----- & ----- & ------------ & ---- \\
\hline \multicolumn{7}{|l|}{ LNphi } \\
\hline duration & .0118 & $.01842^{\prime}$ & 0.65 & 0.519 & -.02 & 46 \\
\hline cons & -.6963666 & .1648696 & -4.22 & 0.000 & -1.019505 & -.3732281 \\
\hline---- & - & -- & . & -1 & --- & --- \\
\hline duration & -.019933 & .0 & -0.50 & 0.617 & -.0 & 68 \\
\hline _cons & .4690769 & .3980416 & 1.18 & 0.239 & -.3110703 & 1. 249224 \\
\hline
\end{tabular}




\begin{tabular}{r|rrrrrr} 
mu duration & .0014873 & .001405 & 1.06 & 0.290 & -.0012664 & .004241 \\
_cons & .0185893 & .0106805 & 1.74 & 0.082 & -.0023442 & .0395228
\end{tabular}

- test duration

( 1) [rRDU] duration $=0$

( 2) [LNeta]duration $=0$

(3) [LNphi]duration $=0$

(4) [kappa]duration $=0$

( 5) [mu] duration $=0$

$$
\begin{array}{rll}
\operatorname{chi} 2(5) & = & 4.10 \\
\text { Prob }>\text { chi2 } & = & 0.5344
\end{array}
$$

- ml model lf ML_eut_rdu_prelec2c (rEUT: choiceR \$Rdata = duration ) (rRDU: duration )

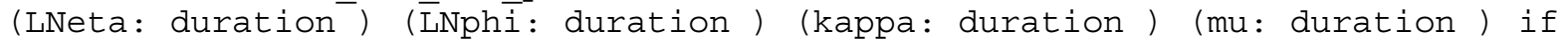
qid_record $==1$, cluster(sid) maximize technique(nr) difficult continue

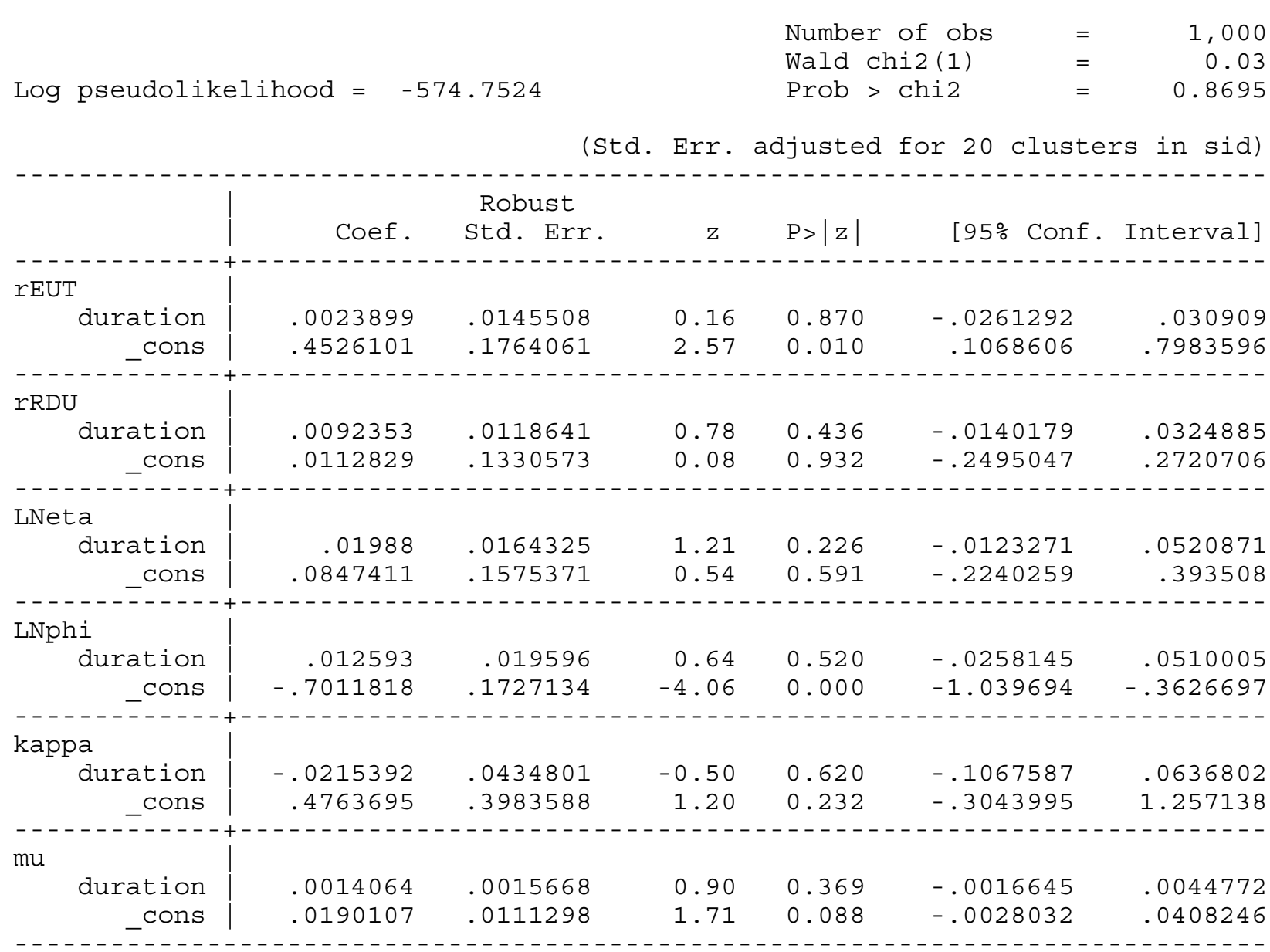

- test duration

(1) $[$ rEUT] duration $=0$

( 2) [rRDU] duration $=0$

(3) [LNeta]duration $=0$

(4) [LNphi]duration $=0$

(5) [kappa]duration $=0$ 
(6) $[\mathrm{mu}]$ duration $=0$

$\begin{aligned} \operatorname{chi}(6)= & 4.31 \\ \text { Prob }>\operatorname{chi2} & =0.6350\end{aligned}$

Estimates of the EUT-RDU Mixture Model with Eye-Tracking and Demographic Covariates

- ml model lf ML_eut_rdu_prelec2c (rEUT: choiceR \$Rdata = \$eyes age ) (rRDU: \$eyes \$demog) (LNeta: \$eyes \$demog) (LNphi: \$eyes \$demog) (kappa: \$eyes \$demog ) (mu: \$eyes ) if qid_record==1, cluster(sid) maximize technique(nr) difficult continue

Log pseudolikelihood $=-536.12719$

$\begin{array}{llr}\text { Number of obs } & = & 1,000 \\ \text { Wald chi2 }(0) & = & \text {. } \\ \text { Prob }>\text { chi2 } & = & .\end{array}$

(Std. Err. adjusted for 20 clusters in sid)

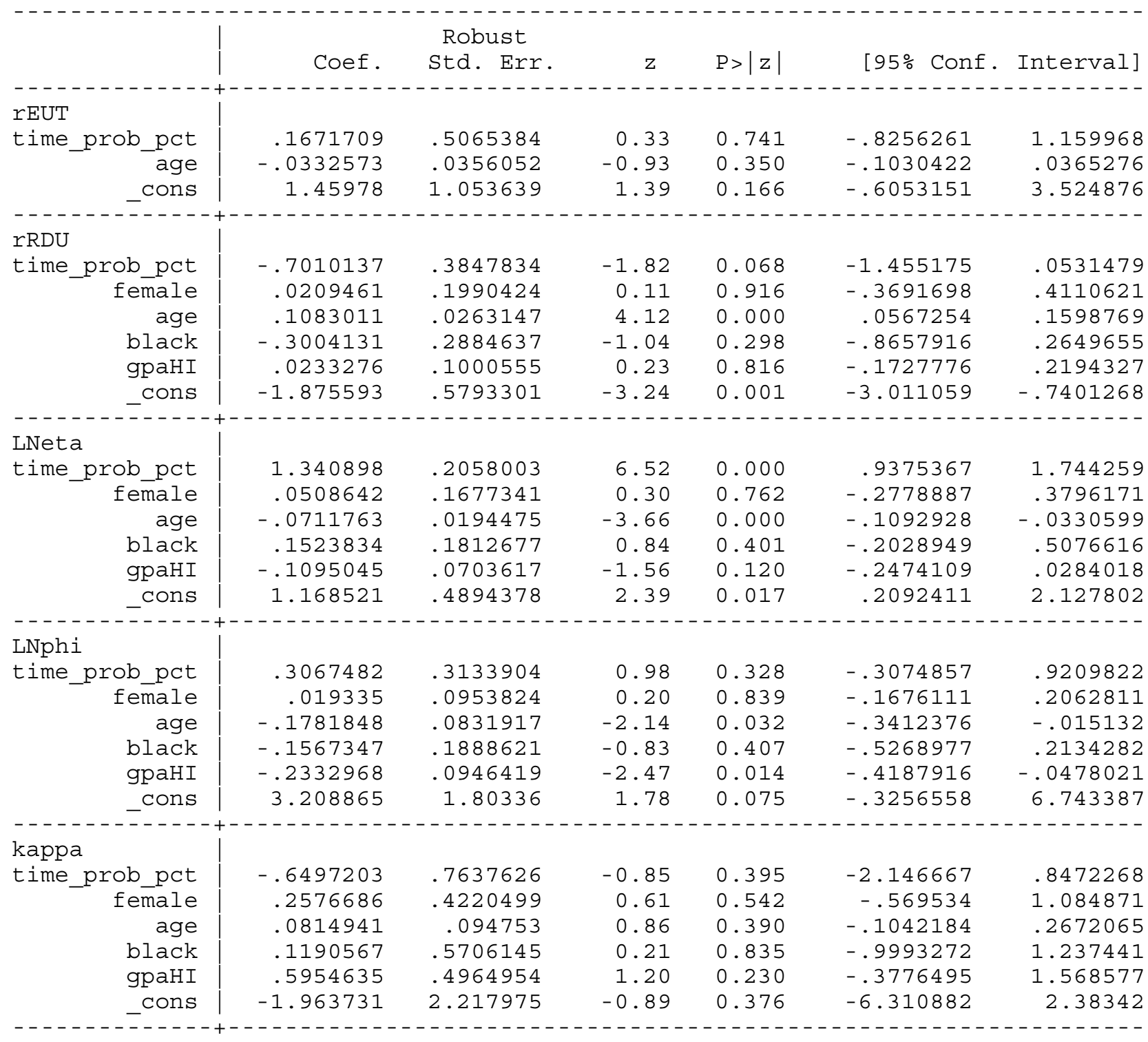




\begin{tabular}{|c|c|c|c|c|c|c|}
\hline $\mathrm{mu}$ & & & & & & \\
\hline time_prob_pct & .0205365 & .0157074 & 1.31 & 0.191 & -.0102494 & .0513225 \\
\hline - Cons & .0032979 & .0041826 & 0.79 & 0.430 & -.0048999 & .0114957 \\
\hline
\end{tabular}

- nlcom (rEUT: [rEUT]_cons) (rRDU: [rRDU]_cons) (eta: exp ([LNeta]_b[_cons])) (phi: $\exp ([$ LNphi]_b[_cons $]))$ (pEUT: 1/(1+exp ([kāppa]_cons $)))$ (mu: [mu]_cons $)$

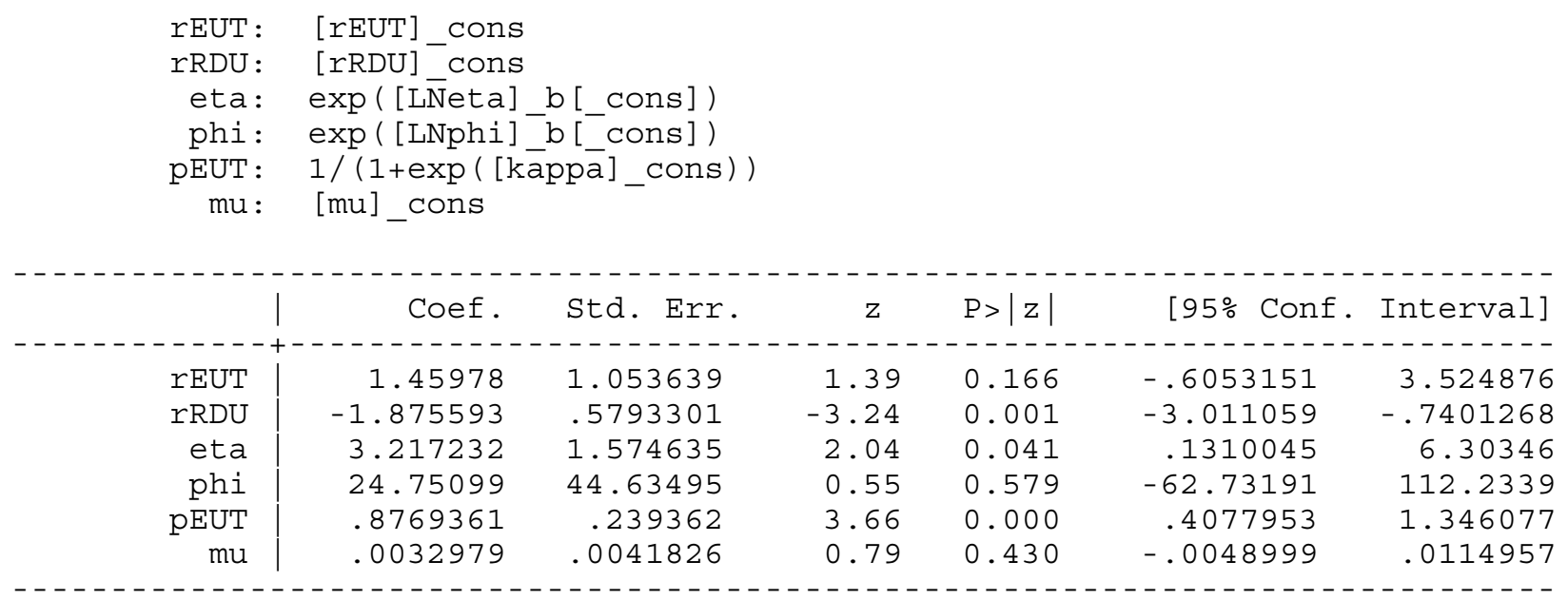

rEUT_time_ t: [rEUT]_cons+[rEUT] time_prob_pct - [rEUT]_cons rRDU_time_ t: [rRDU]_cons+[rRDU] time_prob_pct - [rRDU] ${ }_{-}^{-}$cons eta_time_p t: $\exp ([\mathrm{L} \overline{\mathrm{N}} e t a]$ cons+[LNeta] time_prob_pct) - $\exp ([$ LNeta] cons) phi_time_p t: $\quad \exp ([$ LNphi]_cons+[LNphi]time_prob_pct) - exp ([LNphi]_cons) pEUT_time_ t: $1 /(1+\exp ([\mathrm{kappa}]$ cons + [kappa]time_prob_pct) $)-1 /(\overline{1}+\exp ([\mathrm{kappa}]$ _cons $))$ mu_time_prot: [mu]_cons+[mu]time_prob_pct $-[\mathrm{mu}]$ _cons

\begin{tabular}{|c|c|c|c|c|c|c|}
\hline & Coef & Std. Err. & $\mathrm{z}$ & $P>|z|$ & {$[95 \%$ Conf } & Interval] \\
\hline & & 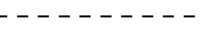 & & 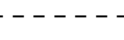 & & ------- \\
\hline rEUT_time_prob_pct & .1671709 & .5065384 & 0.33 & 0.741 & -.8256261 & 1.159968 \\
\hline rRDU_time_prob_pct & -.7010137 & .3847834 & -1.82 & 0.068 & -1.455175 & .0531479 \\
\hline eta_time_prob_pct & 9.080556 & 4.966064 & 1.83 & 0.067 & -.6527495 & 18.81386 \\
\hline phi_time_prob_pct & 8.885575 & 12.62497 & 0.70 & 0.482 & -15.85892 & 33.63007 \\
\hline pEUT_time_prob_pct & .0547861 & .1236918 & 0.44 & 0.658 & -.1876453 & .2972175 \\
\hline mu_time_prob_pct & .0205365 & .0157074 & 1.31 & 0.191 & -.0102494 & .0513225 \\
\hline
\end{tabular}

( 1$)$ [rRDU] time_prob_pct $=0$

$$
\begin{array}{rll}
\operatorname{chi} 2(1) & = & 3.32 \\
\text { Prob }>\text { chi2 } & =0.0685
\end{array}
$$

( 1$)$ [LNeta] time_prob_pct $=0$

( 2) [LNphi]time_prob_pct $=0$

$$
\begin{aligned}
\operatorname{chi} 2(2) & =53.08 \\
\text { Prob }>\text { chi2 } & =0.0000
\end{aligned}
$$

( 1$)$ [rEUT] time_prob_pct $=0$

( 2) [rRDU] time_prob_pct $=0$

( 3 ) [LNeta]time prob_pct $=0$

( 4) [LNphi]time_prob_pct $=0$

( 5) [kappa]time_prob_pct $=0$ 
( 6) [mu]time_prob_pct $=0$

$\begin{array}{rrr}\operatorname{chi}(6) & = & 186.52 \\ \text { Prob }>\operatorname{chi2} & = & 0.0000\end{array}$

eta_age: $\exp ([$ LNeta]_cons+[LNeta]age) - exp ([LNeta]_cons)

phi_age: exp ([LNphi]_Cons+[LNphi]age) - exp ([LNphi]_Cons)

pEUT_age: $1 /(1+\exp ([\mathrm{k}$ appa $]$ cons + [kappa $]$ age $))-1 /(\overline{1}+\exp ([\mathrm{kappa}]$ cons $))$

\begin{tabular}{|c|c|c|c|c|c|c|}
\hline & Coef & Std. Err. & $\mathrm{z}$ & $P>|z|$ & [95\% Conf. & Interval] \\
\hline & & & & & & \\
\hline eta_age & -.2210313 & .1639796 & -1.35 & 0.178 & -.5424254 & .1003627 \\
\hline phi_age & -4.039665 & 8.994961 & -0.45 & 0.653 & -21.66946 & 13 \\
\hline pEUT_age & -.0090683 & .0059729 & -1.52 & 0.129 & -.020775 & 84 \\
\hline & & 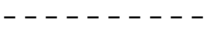 & & & 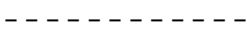 & \\
\hline eta_f & \multicolumn{3}{|c|}{$\exp ([$ LNeta $]$ cons+[LNeta $]$ female $)$} & $-\exp ($ & ta]_cons) & \\
\hline phi_f & \multicolumn{3}{|c|}{$\exp ([\mathrm{LNphi}]$ _cons+[LNphi] female) } & $-\exp ($ & phi]_cons) & \\
\hline pEUT_fe & \multicolumn{6}{|c|}{ 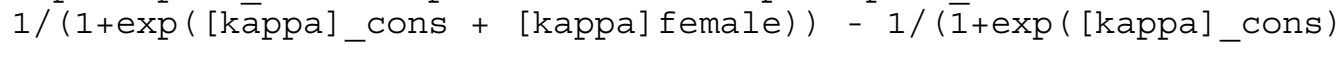 } \\
\hline \multirow{2}{*}{\multicolumn{7}{|c|}{ 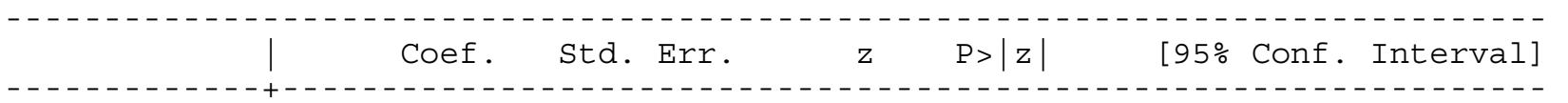 }} \\
\hline & & & & & & \\
\hline eta_fe & .1678751 & 0893 & 0.32 & 0.751 & -.8710809 & 1.2 \\
\hline phi_fe & .4832169 & 2.148132 & 0.22 & 0.822 & -3.727045 & 3478 \\
\hline pEUT_female & -.0306113 & .0709791 & -0.43 & 0.666 & -.1697277 & .1085051 \\
\hline
\end{tabular}

(1) [rRDU] female $=0$

$\begin{array}{rll}\operatorname{chi} 2(1) & = & 0.01 \\ \text { Prob }>\text { chi2 } & =0.9162\end{array}$

( 1$)$ [LNeta] female $=0$

( 2) [LNphi]female $=0$

$\begin{array}{rll}\operatorname{chi2}(2) & = & 0.09 \\ \text { Prob }>\text { chi2 } & =0.9546\end{array}$

( 1$)$ [rRDU] female $=0$

( 2) [LNeta] female $=0$

( 3 ) [LNphi] female $=0$

(4) [kappa] female $=0$

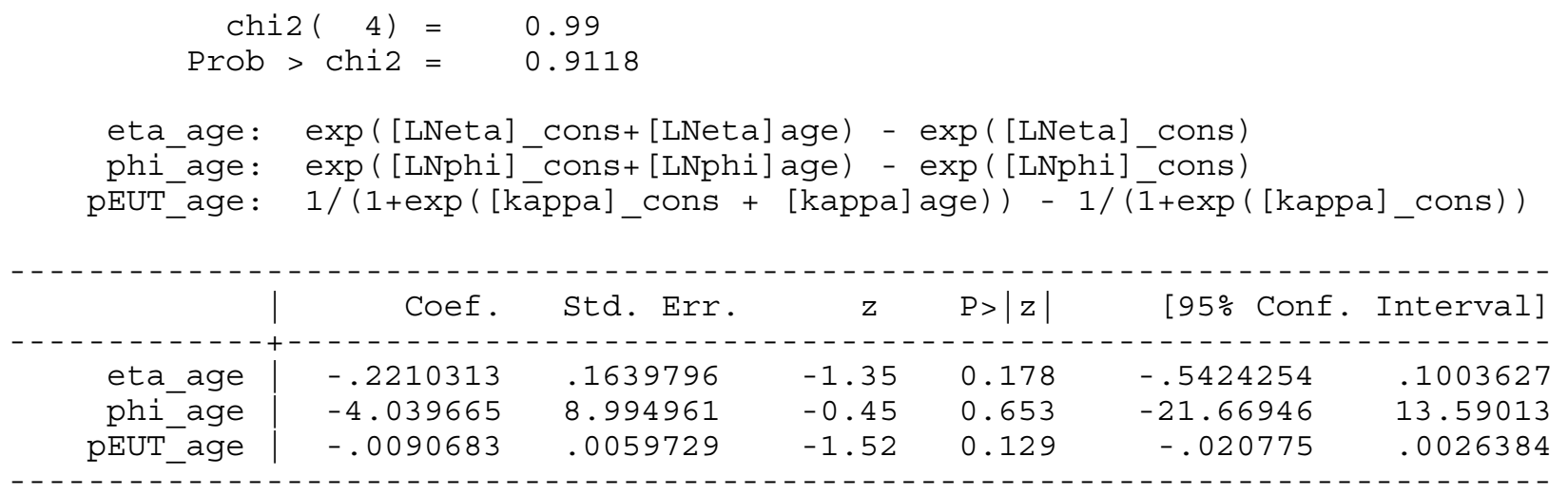


(1) [rRDU] age $=0$

$$
\begin{array}{rlr}
\operatorname{chi} 2(1) & = & 16.94 \\
\text { Prob }>\text { chi2 } & = & 0.0000
\end{array}
$$

( 1) [LNeta] age $=0$

(2) [LNphi] age $=0$

$$
\begin{array}{rlc}
\operatorname{chi} 2(2)= & 14.60 \\
\text { Prob }>\text { chi2 } & 0.0007
\end{array}
$$

(1) [rEUT] age $=0$

(2) [rRDU] age $=0$

(3) [LNeta] age $=0$

(4) [LNphi] age $=0$

( 5) [kappa] age $=0$

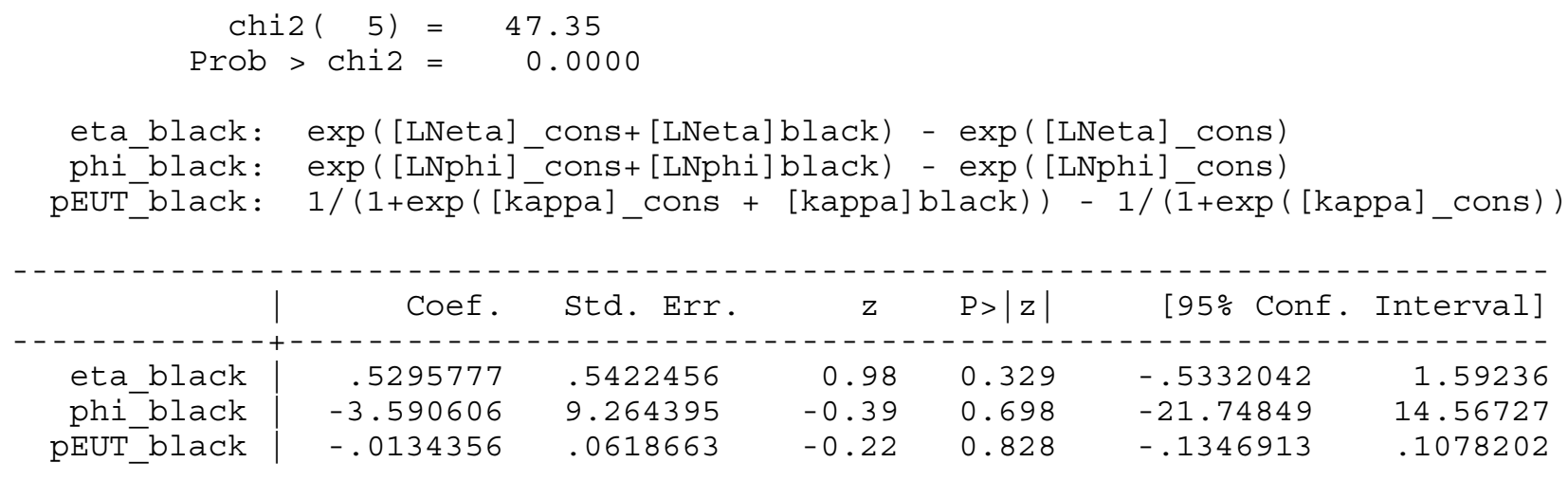

(1) $[r \mathrm{RDU}] \mathrm{black}=0$

$\begin{array}{rll}\operatorname{chi} 2(1) & = & 1.08 \\ \text { Prob }>\text { chi2 } & =0.2977\end{array}$

( 1$)$ [LNeta]black $=0$

( 2) [LNphi]black $=0$

$$
\begin{array}{rll}
\operatorname{chi}(2) & = & 2.45 \\
\text { Prob }>\text { chi2 } & = & 0.2943
\end{array}
$$

( 1$)$ [rRDU]black = 0

( 2) [LNeta]black $=0$

( 3 ) [LNphi]black $=0$

\begin{tabular}{|c|c|c|c|c|c|c|}
\hline & Coef & std. Err. & $z$ & $\mathrm{P}>|\mathrm{z}|$ & [95\% Conf. & Interval] \\
\hline eta_gpaHI & -.3336974 & .2525875 & -1.32 & 0.186 & -.8287599 & .161365 \\
\hline phi_gpaHI & -5.150224 & 8.855219 & -0.58 & 0.561 & -22.50613 & 12.20569 \\
\hline
\end{tabular}

(4) [kappa]black $=0$

$\begin{aligned} \operatorname{chi} 2(4) & =2.87 \\ \operatorname{Prob}>\operatorname{chi} 2 & =0.5791\end{aligned}$

eta_gpaHI: $\exp ([$ LNeta]_cons+[LNeta]gpaHI) - exp ([LNeta]_cons)

phi_gpaHI: exp ([LNphi]_cons+[LNphi]gpaHI) - exp ([LNphi]_cons)

pEUT_gpaHI : $1 /(1+\exp ([\mathrm{kapppa}]$ cons + [kappa]gpaHI $))-1 /(\overline{1}+\exp ([\mathrm{kappa}]$ cons $))$ 


pEUT_gpaHI | -.0798361 .1489559 $-0.54 \quad 0.592 \quad-.3717843 \quad .2121121$

( 1$) \quad[r R D U]$ gpaHI $=0$

$\begin{aligned} \operatorname{chi}(1)= & 0.05 \\ \operatorname{Prob}>\operatorname{chi} 2 & =0.8156\end{aligned}$

( 1 [ [LNeta] gpaHI $=0$

( 2) [LNphi] gpaHI $=0$

$\begin{array}{rll}\operatorname{chi} 2(2) & = & 7.41 \\ \text { Prob }>\text { chi2 } & = & 0.0246\end{array}$

( 1$)$ [rRDU] gpaHI $=0$

(2) [LNeta] gpaHI $=0$

( 3 ) [LNphi] gpaHI $=0$

(4) [kappa] gpaHI $=0$

$$
\begin{array}{rlr}
\operatorname{chi}(4) & =21.68 \\
\text { Prob }>\text { chi2 } & = & 0.0002
\end{array}
$$

\section{Estimates of the EUT-RDU Mixture Model with All Covariates}

- ml model lf ML_eut_rdu_prelec2c (rEUT: choiceR \$Rdata = \$eyes age ) (rRDU: duration \$eyes \$demog) (LNetā: düration \$eyes \$demog) (LNphi: duration \$eyes \$demog) (kappa: duration \$eyes \$demog) (mu: \$eyes) if qid_record==1, cluster(sid) maximize technique (nr) difficult continue

Log pseudolikelihood $=-532.55615$

$\begin{array}{llr}\text { Number of obs } & = & 1,000 \\ \text { Wald chi2 }(0) & = & . \\ \text { Prob > chi2 } & = & .\end{array}$

(Std. Err. adjusted for 20 clusters in sid)

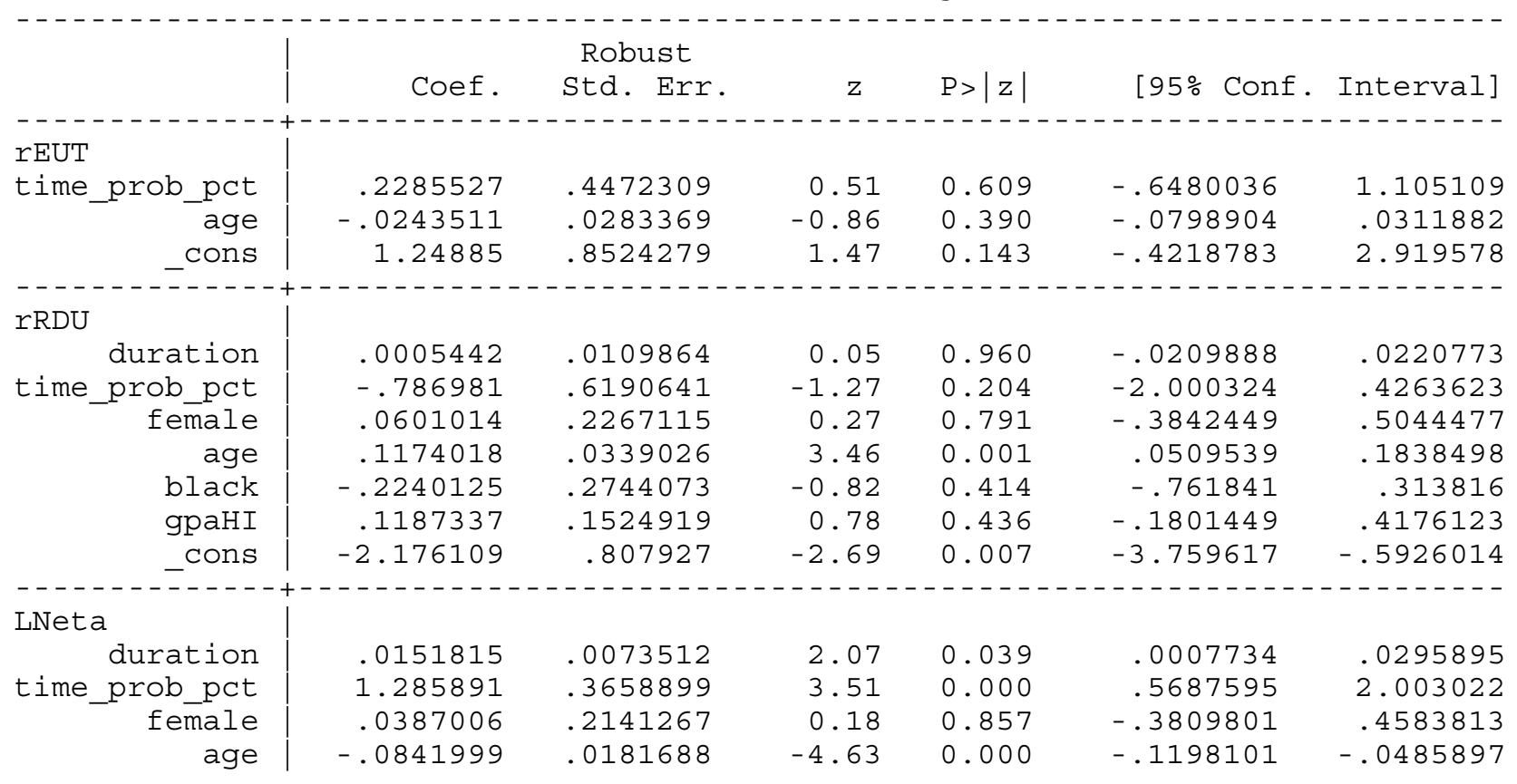

$-\mathrm{A} 30-$ 


\begin{tabular}{|c|c|c|c|c|c|c|}
\hline black & .0640525 & .1708391 & 0.37 & 0.708 & -.2707861 & .3988911 \\
\hline gранI & -.2196714 & .1158941 & -1.90 & 0.058 & -.4468196 & .0074768 \\
\hline Cons & 1.503196 & .416379 & 3.61 & 0.000 & .6871081 & 2.319284 \\
\hline 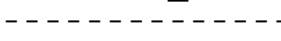 & ---- & ------ & ---- & ----- & -------- & ------- \\
\hline Nphi & & & & & & \\
\hline duration & .0033219 & .015647 & 0.21 & 0.832 & -.0273457 & .0339895 \\
\hline time_prob_pct & .0733387 & .4021538 & 0.18 & 0.855 & -.7148683 & .8615457 \\
\hline female & -.0224462 & .0960092 & -0.23 & 0.815 & -.2106209 & .1657284 \\
\hline age & -.235324 & .0437836 & -5.37 & 0.000 & -.3211383 & -.1495096 \\
\hline black & -.209479 & .150538 & -1.39 & 0.164 & -.504528 & .08557 \\
\hline gpaHI & -.2429425 & .112502 & -2.16 & 0.031 & -.4634425 & -.0224426 \\
\hline cons & 4.461215 & .967804 & 4.61 & 0.000 & 2.564354 & 6.358076 \\
\hline--- & --------- & ------ & ---- & ----- & -------- & -------- \\
\hline kappa & & & & & & \\
\hline duration & -.0321199 & .0264134 & -1.22 & 0.224 & -.0838892 & .0196494 \\
\hline time_prob_pct & -.5787758 & .7251814 & -0.80 & 0.425 & -2.000105 & .8425536 \\
\hline female & .1773933 & .3853461 & 0.46 & 0.645 & -.5778712 & .9326577 \\
\hline age & .0634234 & .0821648 & 0.77 & 0.440 & -.0976167 & .2244635 \\
\hline $\mathrm{black}$ & .1451783 & .5748008 & 0.25 & 0.801 & -.9814106 & 1.271767 \\
\hline gpaHI & .6054713 & .4520069 & 1.34 & 0.180 & -.280446 & 1.491388 \\
\hline cons & -1.385882 & 1.892236 & -0.73 & 0.464 & -5.094597 & 2.322832 \\
\hline & --- & ---- & --- & -- & ------- & ------- \\
\hline & & & & & & \\
\hline time_prob_pct & .0110362 & .022576 & 0.49 & 0.625 & -.0332119 & .0552843 \\
\hline$-\overline{c o n s}$ & .005147 & .0081558 & 0.63 & 0.528 & -.010838 & .0211319 \\
\hline
\end{tabular}

. nlcom (rEUT: [rEUT]_cons) (rRDU: [rRDU]_cons) (eta: exp ([LNeta]_b [_cons])) (phi: $\exp ([$ LNphi]_b[_cons] $))($ pEUT: $1 /(1+\exp ([\mathrm{kappa}]$ cons $)))(\mathrm{mu}:$ [mu]_cons $)$

\begin{tabular}{|c|c|}
\hline rEUT : & [rEUT]_cons \\
\hline rRDU : & [rRDU]_cons \\
\hline eta: & $\exp ([$ LNeta]_b [_cons $])$ \\
\hline phi: & $\exp ([$ LNphi]_b [_cons $])$ \\
\hline $\begin{array}{r}\text { pEUT : } \\
\mathrm{mu}:\end{array}$ & $\begin{array}{l}\text { 1/(1+exp }([k \overline{a p p} \bar{a}] \text { _cons })) \\
\text { [mu]_cons }\end{array}$ \\
\hline
\end{tabular}

\begin{tabular}{|c|c|c|c|c|c|c|}
\hline & Coef. & Std. Err. & $\mathrm{z}$ & $\mathrm{P}>|\mathrm{z}|$ & {$[95 \%$ Conf } & Interval] \\
\hline & ----- & ------- & --- & ----- & ------- & ------- \\
\hline rEUT & 1.24885 & .8524279 & 1.47 & 0.143 & -.4218783 & 2.919578 \\
\hline$r \mathrm{RDU}$ & -2.176109 & .807927 & -2.69 & 0.007 & -3.759617 & -.5926014 \\
\hline eta & 4.496035 & 1.872054 & 2.40 & 0.016 & .8268758 & 8.165194 \\
\hline phi & 86.59267 & 83.80473 & 1.03 & 0.301 & -77.66158 & 250.8469 \\
\hline pEUT & .7999341 & .3028326 & 2.64 & 0.008 & .206393 & 1.393475 \\
\hline mu & .005147 & .0081558 & 0.63 & 0.528 & -.010838 & .0211319 \\
\hline
\end{tabular}

rRDU_durat n : [rRDU]_cons+[rRDU] duration - [rRDU]_cons eta duration: $\exp ([\mathrm{L} \overline{\mathrm{N}} e t a]$ cons+[LNeta]duration) - $\exp ([\mathrm{LNeta}]$ cons)

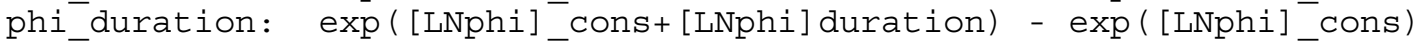
pEUT_durat n: $1 /\left(1+\exp \left([\mathrm{k} \bar{a} p \mathrm{pp}]_{\text {_cons }}+[\mathrm{kappa}]\right.\right.$ duration $\left.)\right)-1 /(\overline{1}+\exp ([\mathrm{kappa}]$ cons $))$

\begin{tabular}{|c|c|c|c|c|c|c|}
\hline & Coef & Std. Err. & z & $\mathrm{P}>|\mathrm{z}|$ & {$[95 \%$ Conf } & Interval] \\
\hline 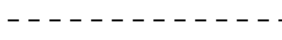 & --- & ------ & 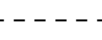 & ---- & ------ & ----- \\
\hline rRDU_duration & .0005442 & .0109864 & 0.05 & 0.960 & -.0209888 & .0220773 \\
\hline eta_duration & .0687771 & .0537863 & 1.28 & 0.201 & -.0366422 & .1741964 \\
\hline phi_duration & .2881307 & 1.432984 & 0.20 & 0.841 & -2.520466 & 3.096728 \\
\hline pEUT_duration & .005091 & .0071355 & 0.71 & 0.476 & -.0088944 & .0190764 \\
\hline
\end{tabular}


(1) [rRDU] duration $=0$

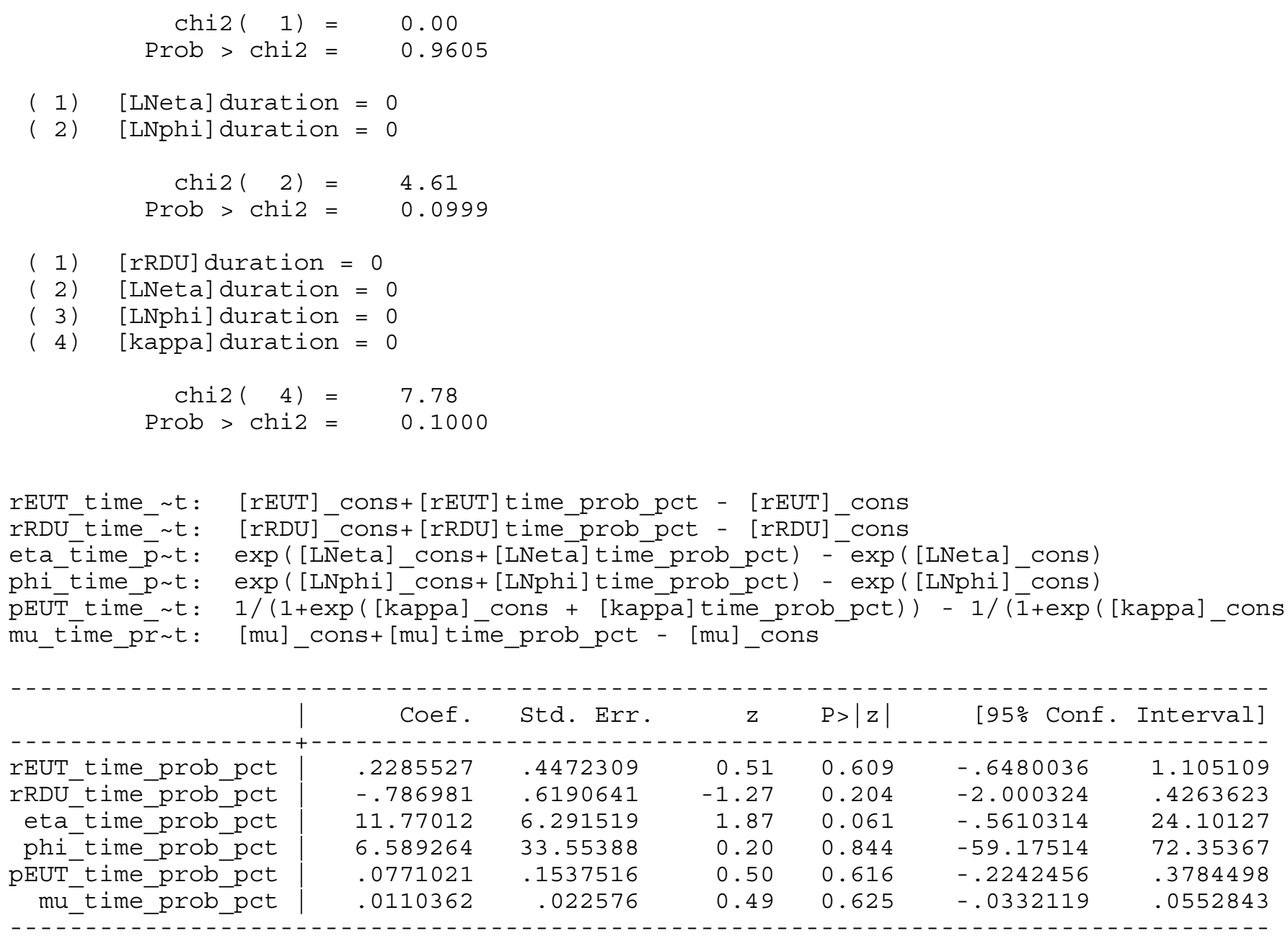

( 1) [rRDU] time_prob_pct $=0$

$\begin{aligned} \operatorname{chi2}(1) & =1.62 \\ \text { Prob }>\text { chi2 } & =0.2036\end{aligned}$

( 1) [LNeta] time_prob_pct $=0$

( 2) [LNphi]time_prob_pct $=0$

$$
\begin{aligned}
\operatorname{chi2}(2) & =21.03 \\
\text { Prob }>\text { chi2 } & =0.0000
\end{aligned}
$$

( 1) [rEUT] time_prob_pct $=0$

( 2) [rRDU] time_prob_pct $=0$

( 3) [LNeta] time prob pct $=0$

( 4) [LNphi]time_prob_pct $=0$

( 5) [kappa]time_prob_pct $=0$

( 6) [mu]time_prob_pct $=0$

$$
\begin{aligned}
\operatorname{chi} 2(6) & =132.44 \\
\text { Prob }>\operatorname{chi} 2 & =0.0000
\end{aligned}
$$




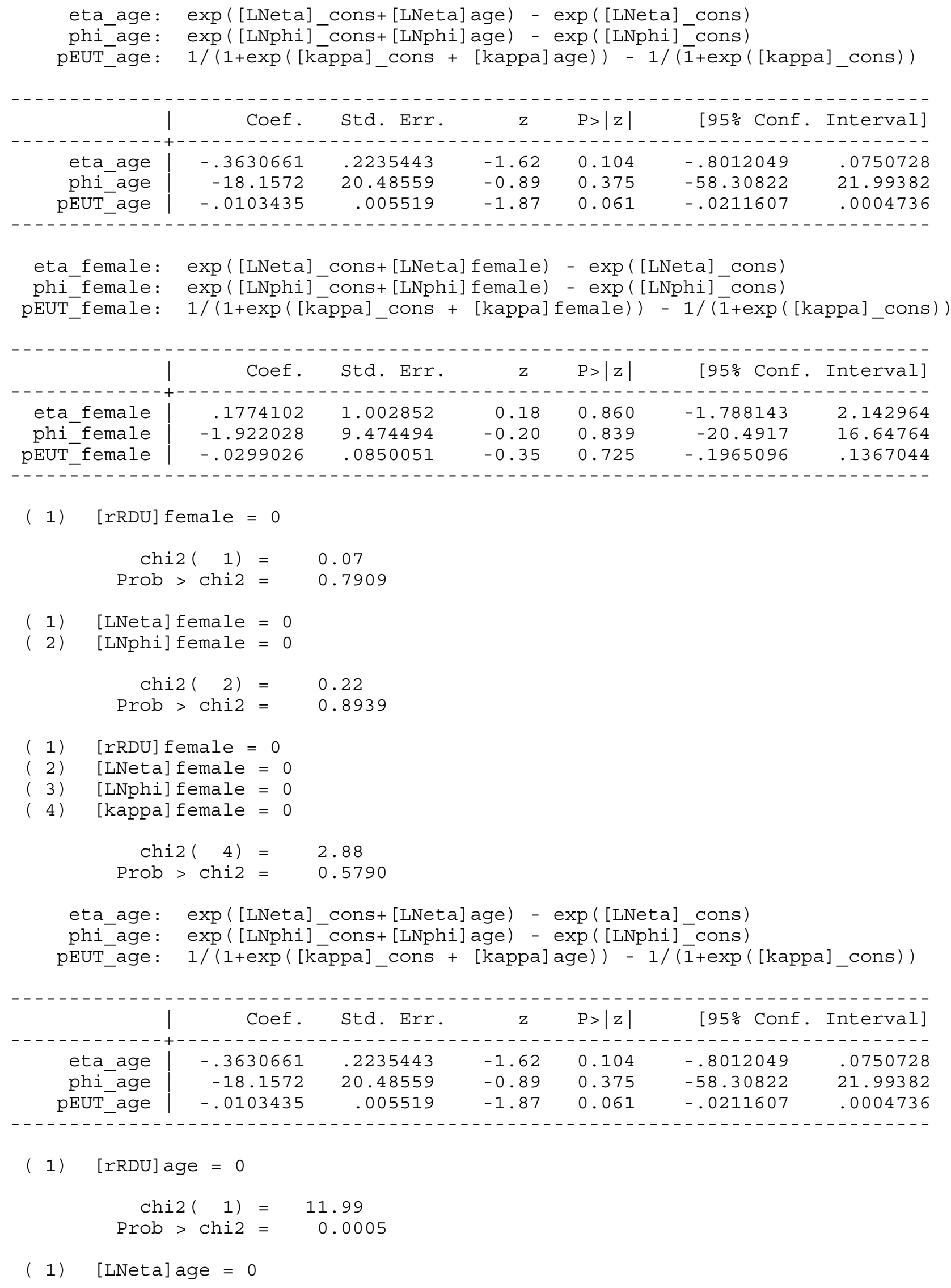


(2) [LNphi] age $=0$

$\begin{aligned} \operatorname{chi} 2(2)= & 39.95 \\ \text { Prob }>\text { chi2 } & 0.0000\end{aligned}$

(1) [rEUT] age $=0$

( 2) [rRDU] age $=0$

(3) [LNeta] age $=0$

(4) [LNphi] age $=0$

(5) [kappa] age $=0$

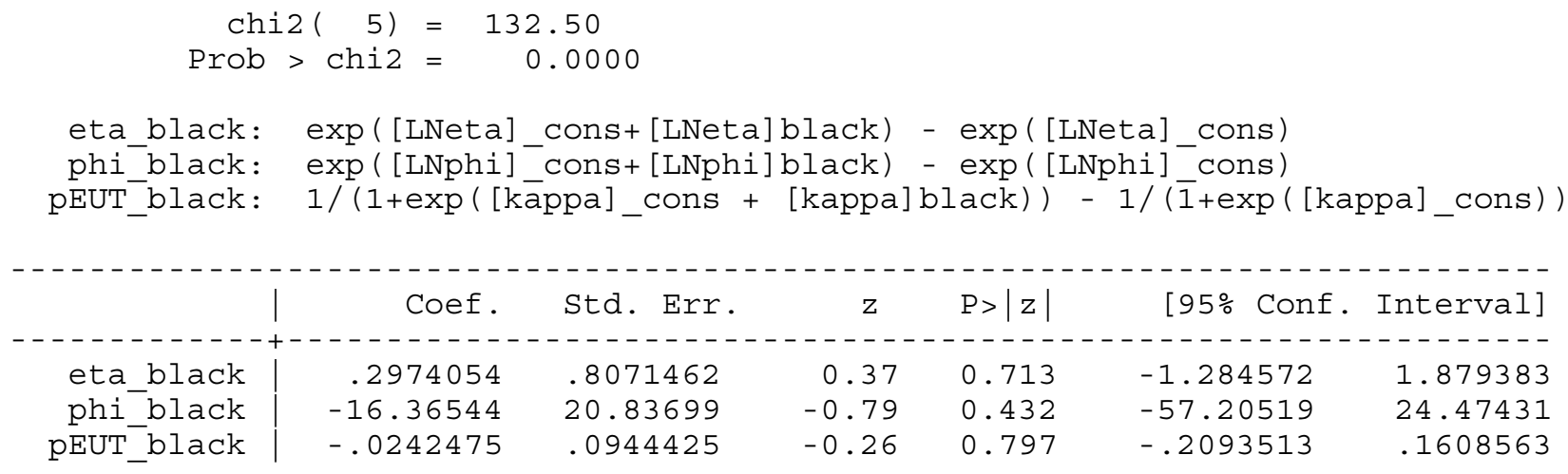

(1) $[r \mathrm{RDU}] \mathrm{black}=0$

$$
\begin{array}{rll}
\operatorname{chi} 2(1) & = & 0.67 \\
\text { Prob }>\text { chi2 } & = & 0.4143
\end{array}
$$

( 1) [LNeta]black $=0$

( 2) [LNphi]black $=0$

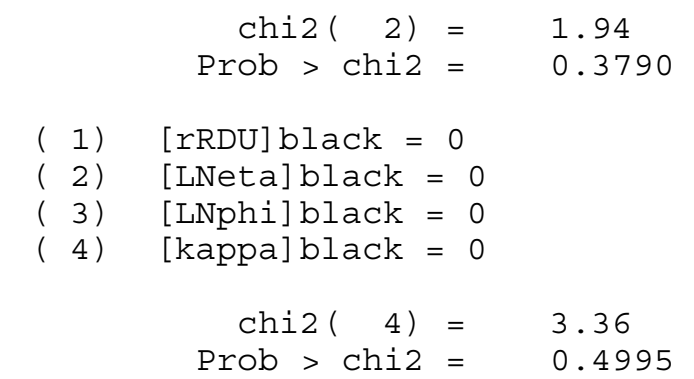

eta gpaHI: $\exp ([$ LNeta]_cons+[LNeta]gpaHI) - exp ([LNeta] cons)

phi_gpaHI: exp ([LNphi]_cons+[LNphi]gpaHI) - exp ([LNphi]_Cons)

\begin{tabular}{|c|c|c|c|c|c|c|}
\hline & Coef & Err. & $z$ & $\mathrm{P}>|\mathrm{z}|$ & [95\% Conf & Interval] \\
\hline------- & ------ & --------- & ----- & ----- & ----------- & -------- \\
\hline eta_gpaHI & -.8866967 & .4413799 & -2.01 & 0.045 & -1.751785 & -.0216079 \\
\hline phi_gpaHI & -18.6766 & 18.29074 & -1.02 & 0.307 & -54.52578 & 17.17258 \\
\hline pEUT_gpaHI & -.1141654 & .1372874 & -0.83 & 0.406 & -.3832437 & .154913 \\
\hline
\end{tabular}

pEUT_gpaHI: $1 /\left(1+\exp \left([\mathrm{k} \overline{a p p} a] \_c o n s+[\mathrm{kappa}]\right.\right.$ gpaHI $\left.)\right)-1 /(\overline{1}+\exp ([\mathrm{kappa}]$ cons $))$

( 1$) \quad[r R D U]$ gpaHI $=0$

$\begin{aligned} \operatorname{chi}(1)= & 0.61 \\ \text { Prob }>\operatorname{chi} 2 & =0.4362\end{aligned}$


( 1) [LNeta] gpaHI $=0$

( 2) [LNphi]gpaHI $=0$

$$
\begin{array}{rlc}
\operatorname{chi} 2(2)= & 15.73 \\
\text { Prob }>\text { chi2 } & 0.0004
\end{array}
$$

( 1$)$ [rRDU] gpaHI $=0$

( 2) [LNeta] gpaHI $=0$

( 3 ) [LNphi]gpaHI $=0$

(4) [kappa] gpaHI $=0$

$$
\operatorname{chi2}(4)=25.49
$$

Prob $>$ chi2 $=0.0000$

$-A 35-$ 